\title{
Iterated stochastic integrals in infinite dimensions: approximation and error estimates
}

\author{
Claudine Leonhard ${ }^{1}$ - Andreas Rößler ${ }^{2}$ (D)
}

Received: 22 September 2017 / Revised: 8 May 2018 / Published online: 22 September 2018

(c) The Author(s) 2018

\begin{abstract}
Higher order numerical schemes for stochastic partial differential equations that do not possess commutative noise require the simulation of iterated stochastic integrals. In this work, we extend the algorithms derived by Kloeden et al. (Stoch Anal Appl 10(4):431441, 1992. https://doi.org/10.1080/07362999208809281) and by Wiktorsson (Ann Appl Probab 11(2):470-487, 2001. https://doi.org/10.1214/aoap/1015345301) for the approximation of two-times iterated stochastic integrals involved in numerical schemes for finite dimensional stochastic ordinary differential equations to an infinite dimensional setting. These methods clear the way for new types of approximation schemes for SPDEs without commutative noise. Precisely, we analyze two algorithms to approximate two-times iterated integrals with respect to an infinite dimensional $Q$-Wiener process in case of a trace class operator $Q$ given the increments of the $Q$ Wiener process. Error estimates in the mean-square sense are derived and discussed for both methods. In contrast to the finite dimensional setting, which is contained as a special case, the optimal approximation algorithm cannot be uniquely determined but is dependent on the covariance operator $Q$. This difference arises as the stochastic process is of infinite dimension.
\end{abstract}

Keywords Iterated stochastic Itô integral in infinite dimensions $\cdot Q$-Wiener process . Lévy area simulation - Karhunen-Loève expansion · Fourier series expansion · Numerical approximation · Error bound

Mathematics Subject Classification $60 \mathrm{H} 05 \cdot 60 \mathrm{H} 15 \cdot 60 \mathrm{H} 35 \cdot 65 \mathrm{C} 30$

$凶 \quad$ Andreas Rößler

roessler@math.uni-luebeck.de

Claudine Leonhard

leonhard@math.uni-kiel.de

1 Department of Mathematics, Christian-Albrechts-Universität zu Kiel, Christian-Albrechts-Platz 4, 24118 Kiel, Germany

2 Institute of Mathematics, Universität zu Lübeck, Ratzeburger Allee 160, 23562 Lübeck, Germany 


\section{Introduction}

In order to obtain higher convergence rates for numerical schemes for stochastic differential equations, in general, we need to incorporate the information contained in iterated integrals. However, usually these integrals cannot be simulated directly. Therefore, we need to replace these terms by an approximation. We illustrate this statement in a finite dimensional setting first, although, we are concerned about the approximation of iterated Itô integrals in infinite dimensions in this work.

The numerical approximation of stochastic ordinary differential equations (SDEs) that do not possess commutative noise requires the simulation of iterated stochastic integrals to achieve a high order of convergence, see $[3,5,9]$. One example of such a higher order scheme is the Milstein scheme developed in [7], which we present below to illustrate the issue. For some fixed $d, K \in \mathbb{N}$, we consider a $d$-dimensional SDE of type

$$
\mathrm{d} X_{t}=a\left(X_{t}\right) \mathrm{d} t+\sum_{j=1}^{K} b^{j}\left(X_{t}\right) \mathrm{d} \beta_{t}^{j}
$$

with functions $a: \mathbb{R}^{d} \rightarrow \mathbb{R}^{d}, b^{j}=\left(b^{1, j}, \ldots, b^{d, j}\right)^{T}: \mathbb{R}^{d} \rightarrow \mathbb{R}^{d}, j \in\{1, \ldots, K\}$ for all $t \geq 0$ and initial value $X_{0}=x_{0} \in \mathbb{R}^{d}$. Moreover, $\left(\beta_{t}^{j}\right)_{t \geq 0}, j \in\{1, \ldots, K\}$ denote independent real-valued Brownian motions. For some $T>0$, we divide the time interval $[0, T]$ into $M \in \mathbb{N}$ equal time steps $h=\frac{T}{M}$ and denote $t_{m}=m h$ for $m \in$ $\{0, \ldots, M\}$. The increments of the Brownian motion are given as $\Delta \beta_{m}^{j}=\beta_{t_{m+1}}^{j}-\beta_{t_{m}}^{j}$ for all $j \in\{1, \ldots, K\}$ and $m \in\{0, \ldots, M-1\}$. Then, the Milstein scheme [7] reads as $Y_{0}=x_{0}$ and

$$
\begin{aligned}
Y_{m+1}= & Y_{m}+a\left(Y_{m}\right) h+\sum_{j=1}^{K} b^{j}\left(Y_{m}\right) \Delta \beta_{m}^{j} \\
& +\sum_{i, j=1}^{K}\left(\frac{\partial b^{l, i}}{\partial x_{k}}\left(Y_{m}\right)\right)_{1 \leq l, k \leq d} b^{j}\left(Y_{m}\right) \int_{t_{m}}^{t_{m+1}} \int_{t_{m}}^{s} \mathrm{~d} \beta_{r}^{i} \mathrm{~d} \beta_{s}^{j}
\end{aligned}
$$

for $m \in\{0, \ldots, M-1\}$ using the notation $Y_{m}=Y_{t_{m}}$. Under suitable assumptions, the following error estimate holds

$$
\left(\mathrm{E}\left[\left|X_{T}-Y_{M}\right|^{2}\right]\right)^{\frac{1}{2}} \leq C h
$$

see [3]. If SDE (1) does not possess commutative noise, see [3] for details, the Milstein scheme cannot be simplified and one has to approximate the iterated stochastic integrals involved in the method. We denote these iterated Itô integrals by

$$
I_{(i, j)}(h)=I_{(i, j)}(t, t+h):=\int_{t}^{t+h} \int_{t}^{s} \mathrm{~d} \beta_{r}^{i} \mathrm{~d} \beta_{s}^{j}
$$


for some $t \geq 0, h>0$, and for all $i, j \in\{1, \ldots, K\}$, where $K \in \mathbb{N}$ is the number of independent Brownian motions driving the SDE. The research by Kloeden, Platen and Wright [4] and by Wiktorsson [9] suggests different methods for an approximation of these integrals; the main ideas are outlined in Sect. 2. We denote by $\bar{I}_{(i, j)}^{(D)}(h)$ the approximation of $I_{(i, j)}(h)$ with the algorithm derived in [4] for $i, j \in\{1, \ldots, K\}$, $D, K \in \mathbb{N}, h>0$. In [4], the authors proved that for all $i, j \in\{1, \ldots, K\}$ and $h>0$, it holds

$$
\mathrm{E}\left[\left|I_{(i, j)}(h)-\bar{I}_{(i, j)}^{(D)}(h)\right|^{2}\right] \leq C \frac{h^{2}}{D},
$$

where $D \in \mathbb{N}$ denotes the index of the summand at which the series representation of the stochastic double integral is truncated to obtain the approximation $\bar{I}_{(i, j)}^{(D)}(h)$. If we use the algorithm derived in [9] instead, we denote the approximation of $I_{(i, j)}(h)$ by $\hat{I}_{(i, j)}^{(D)}(h)$ for all $i, j \in\{1, \ldots, K\}, h>0$. This scheme employs the same series representation as proposed in [4] but incorporates an approximation of the truncated term additionally. The error resulting from this scheme is estimated as

$$
\sum_{\substack{i, j=1 \\ i<j}}^{K} \mathrm{E}\left[\left|I_{(i, j)}(h)-\hat{I}_{(i, j)}^{(D)}(h)\right|^{2}\right] \leq \frac{5 h^{2}}{24 \pi^{2} D^{2}} K^{2}(K-1),
$$

where $D$ is again the index of the summand at which the series is truncated to obtain the approximation and $K$ is the number of independent Brownian motions, see [9]. Let $h$ and $K$ be fixed, then both approximations converge in the mean-square sense as $D$ goes to infinity-with a different order of convergence, however. For the numerical approximation of SDEs, the integer $D$ is determined such that the overall order of convergence in the time step is not distorted. For the Milstein scheme, for example, error estimate (2) is considered, that is, a strong order of convergence of 1 can be achieved. Therefore, $D \geq \frac{C}{h}$ is chosen for the method derived in [4], whereas $D \geq$ $\frac{\sqrt{5 K^{2}(K-1)}}{\sqrt{24 \pi^{2} h}}$ is selected for the algorithm developed in [9], see also [3, Cor. 10.6.5]. This shows that if we decrease the step size $h$, the value for $D$ has to increase faster for the scheme developed in [4]. Note that the error estimate (4) depends on the number of Brownian motions $K$ as well. As this number is fixed in the setting of finite dimensional SDEs, this factor is not crucial but simply a constant. Therefore, the algorithm proposed by Wiktorsson [9] is superior to the one derived in [4] in terms of the computational effort when a given order of convergence in the step size $h$ is to be achieved.

The same issue arises in the context of higher order numerical schemes designed for infinite dimensional stochastic differential equations that need not have commutative noise-we also have to approximate the involved iterated stochastic integrals in order to implement the scheme. This time, however, the stochastic process is infinite dimensional, in general. In this work, we aim at devising numerical algorithms for the simulation of iterated integrals which arise, for example, in the approximation of the mild solution of stochastic partial differential equations (SPDEs) of type 


$$
\mathrm{d} X_{t}=\left(A X_{t}+F\left(X_{t}\right)\right) \mathrm{d} t+B\left(X_{t}\right) \mathrm{d} W_{t}, \quad t \in(0, T], \quad X_{0}=\xi
$$

where the commutativity condition from [2]

$$
B^{\prime}(y)(B(y) u, v)=B^{\prime}(y)(B(y) v, u)
$$

for all $y \in H_{\beta}, u, v \in U_{0}$ is not assumed to hold. Here, $H_{\beta}=\mathcal{D}\left((-A)^{\beta}\right)$ denotes the domain of the operator $(-A)^{\beta}$ which is a separable Hilbert space for some $\beta \in[0,1)$. The operators $A, F, B$, and the initial value $\xi$ are assumed to fulfill the conditions imposed for the existence of a unique mild solution, see [1], and are not specified further. The spaces are introduced in Sect. 2 and $\left(W_{t}\right)_{t \geq 0}$ denotes a $Q$-Wiener process taking values in some separable Hilbert space $U$ for some trace class operator $Q$. In order to approximate the mild solution of SPDEs of type (5) with a higher order scheme, we need to simulate iterated stochastic integrals of the form

$$
\int_{t}^{t+h} \Psi\left(\int_{t}^{s} \Phi \mathrm{d} W_{r}\right) \mathrm{d} W_{S}
$$

for $t \geq 0, h>0$, and some operators $\Psi, \Phi$ specified in Sect. 2. These terms arise if condition (6) is not fulfilled, for example, in the Milstein scheme for SPDEs [2]. In the Milstein scheme, it holds $\Psi=B^{\prime}\left(Y_{t}\right)$ and $\Phi=B\left(Y_{t}\right)$ for some $B: H \rightarrow$ $L_{H S}\left(U_{0}, H\right)$ and an approximation $Y_{t} \in H_{\beta}$ with $t \geq 0$ and $\beta \in[0,1)$, where $L_{H S}\left(U_{0}, H\right)$ denotes the space of Hilbert-Schmidt operators from $U_{0}$ to $H$. For more details, we refer to [2].

We want to emphasize that the algorithms developed for the approximation of iterated stochastic integrals in the setting of SDEs are designed for some fixed finite number $K$ of driving Brownian motions and that the approximation error (4) even involves this number $K$ as a constant. In contrast, when approximating the solution of SPDEs driven by an infinite dimensional $Q$-Wiener process, this number corresponds to the dimension of the finite-dimensional approximation subspace onto which the $Q$-Wiener process is projected. Thus, the dimension $K$ of the approximation subspace has to increase, in general, to attain higher accuracy, i.e., $K$ is not constant anymore; see the error estimate of the Milstein scheme for SPDEs in [2], for example. Therefore, this aspect has to be taken into account in order to identify an appropriate approximation algorithm. In the following, we derive two algorithms for the approximation of iterated integrals of type (7) based on the methods developed for the finite dimensional setting by Kloeden, Platen, and Wright [4] and by Wiktorsson [9] as well as on [5] for the infinite dimensional case. These algorithms allow for the first time to implement higher order schemes for SPDEs that do not possess commutative noise and include the algorithms that can be used for finite dimensional SDEs as a special case. We show that the method that is superior in the setting of an infinite dimensional $Q$-Wiener process cannot be uniquely determined in general but is dependent on the covariance operator $Q$. In the analysis of the approximation error, we need to incorporate the eigenvalues of the covariance operator $Q$. For the algorithm based on the approach by Kloeden, Platen, and Wright [4], we obtain a similar estimate as in (3) in the mean-square sense, see Corollary 1. For the method derived in the work of Wiktorsson [9], we can prove 
two differing error estimates for the case of infinite dimensional $Q$-Wiener processes by different means. One is the same, apart from constants, as estimate (4). Moreover, the fact that we integrate with respect to a $Q$-Wiener process with a trace class operator $Q$ allows for an alternative proof to the one given in [9]. The result indicates a possibly superior convergence in $K$-this depends on the rate of decay of the eigenvalues of $Q$. Details can be found in Theorem 3 and Theorem 4.

\section{Approximation of iterated stochastic integrals}

Throughout this work, we fix the following setting and notation. Let $H$ and $U$ be separable real-valued Hilbert spaces. In the following, let $\left(\Omega, \mathcal{F}, P,\left(\mathcal{F}_{t}\right)_{t \geq 0}\right)$ be a probability space, let $\left(W_{t}\right)_{t \geq 0}$ denote a $U$-valued $Q$-Wiener process with respect to $\left(\mathcal{F}_{t}\right)_{t \geq 0}$ where $Q$ is a trace class covariance operator, and let $U_{0}:=Q^{1 / 2} U$. Let $L(U, H)$ denote the space of linear and bounded operators mapping from $U$ to $H$. We define $L(U, H)_{U_{0}}:=\left\{\left.T\right|_{U_{0}}: T \in L(U, H)\right\}$ which is a dense subset of the space of Hilbert-Schmidt operators $L_{H S}\left(U_{0}, H\right)$ [8]. Moreover, we assume that the operators $\Phi$ and $\Psi$ in (7) fulfill

(A1) $\Phi \in L(U, H)_{U_{0}}$ with $\left\|\Phi Q^{-\alpha}\right\|_{L_{H S}\left(U_{0}, H\right)}<C$,

(A2) $\Psi \in L\left(H, L\left(Q^{-\alpha} U, H\right)_{U_{0}}\right)$

for some $\alpha \in(0, \infty)$. The parameter $\alpha$ determines the rate of convergence for the approximation of the $Q$-Wiener process, see Theorems 1 or 3 . Note that assumption (A1), needed to prove the convergence of the approximation algorithms for iterated integrals in Theorems 1, 3, and 4, is less restrictive than the condition imposed on the operator $B$ in SPDE (5) to obtain the error estimate for some numerical scheme to approximate its mild solution, e.g., in [6]. However, for the Milstein scheme in [2], assumption (A2) does not need to be fulfilled for the error analysis of the Milstein scheme to hold true.

If we are interested in the approximation of the mild solution of (5), a combination of the error estimate for a numerical scheme to obtain this process and the error from the approximation of the iterated integrals has to be analyzed. In this case, we impose the following assumptions instead

(B1) $\Phi \in L(U, H)_{U_{0}}$,

(B2) $\Psi \in L\left(H, L(U, H)_{U_{0}}\right)$.

For the convergence results in this case, we refer to Corollary 1 and 2, which have to be combined with estimates on the respective numerical scheme. These weaker conditions are sufficient as in the proof the $Q$-Wiener process is approximated first and then the iterated integral is compared to the approximation.

Let $Q \in L(U)$ be a nonnegative and symmetric trace class operator with eigenvalues $\eta_{j}$ and corresponding eigenfunctions $\tilde{e}_{j}$ for $j \in \mathcal{J}$ where $\mathcal{J}$ is some countable index set. The eigenfunctions $\left\{\tilde{e}_{j}: j \in \mathcal{J}\right\}$ constitute an orthonormal basis of $U$, see [8, Prop. 2.1.5]. Then, for the $Q$-Wiener process $\left(W_{t}\right)_{t \geq 0}$, the following series representation holds, see [8, Prop. 2.1.10], 


$$
W_{t}=\sum_{j \in \mathcal{J}}\left\langle W_{t}, \tilde{e}_{j}\right\rangle_{U} \tilde{e}_{j}, \quad t \geq 0
$$

As the $Q$-Wiener process $\left(W_{t}\right)_{t \geq 0}$ is an infinite dimensional stochastic process, it has to be projected to some finite dimensional subspace by truncating the series (8) such that it can be simulated in a numerical scheme. For $K \in \mathbb{N}$, we denote by $\left(W_{t}^{K}\right)_{t \geq 0}$ the projected $Q$-Wiener process, which is defined as

$$
W_{t}^{K}=\sum_{j \in \mathcal{J}_{K}}\left\langle W_{t}, \tilde{e}_{j}\right\rangle_{U} \tilde{e}_{j}, \quad t \geq 0
$$

for some finite index set $\mathcal{J}_{K} \subset \mathcal{J}$ with $\left|\mathcal{J}_{K}\right|=K$. This expression allows to write the iterated integral with respect to the projected $Q$-Wiener process $\left(W_{t}^{K}\right)_{t \geq 0}$ for any $t \geq 0$ and $h>0$ as

$$
\begin{aligned}
\int_{t}^{t+h} \Psi\left(\int_{t}^{s} \Phi \mathrm{d} W_{r}^{K}\right) \mathrm{d} W_{s}^{K} & =\sum_{i, j \in \mathcal{J}_{K}} \int_{t}^{t+h} \Psi\left(\int_{t}^{s} \Phi\left\langle\mathrm{d} W_{r}, \tilde{e}_{i}\right\rangle_{U} \tilde{e}_{i}\right)\left\langle\mathrm{d} W_{s}, \tilde{e}_{j}\right\rangle_{U} \tilde{e}_{j} \\
& =\sum_{i, j \in \mathcal{J}_{K}} I_{(i, j)}^{Q}(t, t+h) \Psi\left(\Phi \tilde{e}_{i}, \tilde{e}_{j}\right)
\end{aligned}
$$

with

$$
I_{(i, j)}^{Q}(t, t+h):=\int_{t}^{t+h} \int_{t}^{s}\left\langle\mathrm{~d} W_{r}, \tilde{e}_{i}\right\rangle_{U}\left\langle\mathrm{~d} W_{s}, \tilde{e}_{j}\right\rangle_{U}
$$

for $i, j \in \mathcal{J}_{K}$. Therefore, we aim at devising a method to approximate the iterated stochastic integrals $I_{(i, j)}^{Q}(t, t+h)$ for all $i, j \in \mathcal{J}_{K}$. Below, we introduce two such algorithms and analyze as well as discuss their convergence properties. For simplicity of notation, we assume, without loss of generality, $\mathcal{J}_{K}=\{1, \ldots, K\}$ with $\eta_{j} \neq 0$ for $j \in \mathcal{J}_{K}$ and denote $I_{(i, j)}^{Q}(h)=I_{(i, j)}^{Q}(t, t+h)$.

\subsection{Algorithm 1}

In the following, we mainly adapt the method introduced by Kloeden, Platen, and Wright [4] to the setting of infinite dimensional stochastic processes. Here, we additionally have to take into account the error arising from the projection of the $Q$-Wiener process to a finite dimensional subspace.

For some $t \geq 0$, the coefficients of the $Q$-Wiener process $w_{t}^{j}:=\left\langle W_{t}, \tilde{e}_{j}\right\rangle_{U}$ are independent real-valued random variables that are $N\left(0, \eta_{j} t\right)$ distributed for $j \in \mathcal{J}$. Thus, the increments $\Delta w_{h}^{j}:=\left\langle W_{t+h}-W_{t}, \tilde{e}_{j}\right\rangle_{U}$ can be easily simulated since $\Delta w_{h}^{j}$ is $N\left(0, \eta_{j} h\right)$ distributed for $j \in \mathcal{J}$ and $h \geq 0$. Our goal is to obtain an approximation of the iterated integrals $I_{(i, j)}^{Q}(h)$ for all $i, j \in \mathcal{J}_{K}, K \in \mathbb{N}, h>0$ given the realizations of the increments $\Delta w_{h}^{j}$ for $j \in \mathcal{J}_{K}$. The following derivation of the approximation method follows the representation in [4] closely. Below, let $K \in \mathbb{N}$ be arbitrarily fixed 
and let us introduce the scaled Brownian bridge process $\left(w_{s}^{j}-\frac{s}{h} w_{h}^{j}\right)_{0 \leq s \leq h}$ for $j \in \mathcal{J}_{K}$ and some $h \in(0, T]$. We consider its series expansion

$$
w_{s}^{j}-\frac{s}{h} w_{h}^{j}=\frac{1}{2} a_{0}^{j}+\sum_{r=1}^{\infty}\left(a_{r}^{j} \cos \left(\frac{2 r \pi s}{h}\right)+b_{r}^{j} \sin \left(\frac{2 r \pi s}{h}\right)\right)
$$

which converges in $L^{2}(\Omega)$. The coefficients are given by the following expressions

$$
\begin{aligned}
& a_{r}^{j}=\frac{2}{h} \int_{0}^{h}\left(w_{u}^{j}-\frac{u}{h} w_{h}^{j}\right) \cos \left(\frac{2 r \pi u}{h}\right) \mathrm{d} u, \\
& b_{r}^{j}=\frac{2}{h} \int_{0}^{h}\left(w_{u}^{j}-\frac{u}{h} w_{h}^{j}\right) \sin \left(\frac{2 r \pi u}{h}\right) \mathrm{d} u
\end{aligned}
$$

for all $j \in \mathcal{J}_{K}, r \in \mathbb{N}_{0}$, and all $0 \leq s \leq h \leq T$, see also [4]. All coefficients $a_{r}^{j}$ and $b_{r}^{j}$ are independent and $N\left(0, \frac{\eta_{j} h}{2 \pi^{2} r^{2}}\right)$ distributed for $r \in \mathbb{N}$ and $j \in \mathcal{J}_{K}$ and it holds $a_{0}^{j}=-2 \sum_{r=1}^{\infty} a_{r}^{j}$. In contrast to [4], the distributions of the coefficients additionally depend on the eigenvalues $\eta_{j}$ of the covariance operator $Q$. In order to obtain an approximation of the scaled Brownian motion $\left(w_{s}^{j}\right)_{0 \leq s \leq h}$ for some $h \in(0, T]$, we truncate expression (10) at some integer $R \in \mathbb{N}$ and define

$$
w_{s}^{j(R)}=\frac{s}{h} w_{h}^{j}+\frac{1}{2} a_{0}^{j}+\sum_{r=1}^{R}\left(a_{r}^{j} \cos \left(\frac{2 r \pi s}{h}\right)+b_{r}^{j} \sin \left(\frac{2 r \pi s}{h}\right)\right) .
$$

In fact, we are interested in the integration with respect to this process. According to Wong and Zakai [10,11], or [3, Ch. 6.1], an integral with respect to process (11) converges to a Stratonovich integral $J(h)$ as $R \rightarrow \infty$. We are, however, interested in the Itô stochastic integral. Following [3, p. 174], the Stratonovich integral $J_{(i, j)}^{Q}(h)$ can be converted to an Itô integral $I_{(i, j)}^{Q}(h), i, j \in \mathcal{J}_{K}$ according to

$$
I_{(i, j)}^{Q}(h)=J_{(i, j)}^{Q}(h)-\frac{1}{2} h \eta_{i} \mathbb{1}_{i=j}
$$

That is, $I_{(i, j)}^{Q}(h)=J_{(i, j)}^{Q}(h)$ for all $i, j \in \mathcal{J}_{K}$ with $i \neq j$. Moreover, we compute

$$
I_{(i, i)}^{Q}(h)=\frac{\left(\Delta w_{h}^{i}\right)^{2}-\eta_{i} h}{2}
$$

directly for $i \in \mathcal{J}_{K}$, see [3, p. 171]. This implies that we only have to approximate $I_{(i, j)}^{Q}(h)$ for $i, j \in \mathcal{J}_{K}$ with $i \neq j$. Thus, we obtain the desired approximation of the Itô stochastic integral directly by integrating with respect to process (11). Without loss of generality, let $t=0$. By (10) and the fact that $\int_{0}^{h} f(u) \mathrm{d} w_{u}^{j}=f(h) w_{h}^{j}-$ $\int_{0}^{h} f^{\prime}(u) w_{u}^{j} \mathrm{~d} u$ for a continuously differentiable function $f:[0, h] \rightarrow \mathbb{R}, h>0$, see 
[3, p. 89], we obtain the following expression for the iterated stochastic integrals for all $i, j \in \mathcal{J}_{K}, i \neq j$, and $h>0$

$$
\begin{aligned}
I_{(i, j)}^{Q}(h)= & \int_{0}^{h} w_{u}^{i} \mathrm{~d} w_{u}^{j} \\
= & \int_{0}^{h}\left(\frac{u}{h} w_{h}^{i}+\frac{1}{2} a_{0}^{i}+\sum_{r=1}^{\infty}\left(a_{r}^{i} \cos \left(\frac{2 r \pi u}{h}\right)+b_{r}^{i} \sin \left(\frac{2 r \pi u}{h}\right)\right)\right) \mathrm{d} w_{u}^{j} \\
= & \frac{w_{h}^{i}}{h}\left(h w_{h}^{j}-\int_{0}^{h} w_{u}^{j} \mathrm{~d} u\right)+\frac{1}{2} a_{0}^{i} w_{h}^{j} \\
& +\sum_{r=1}^{\infty}\left(a_{r}^{i}\left(w_{h}^{j}+\int_{0}^{h} \frac{2 r \pi}{h} \sin \left(\frac{2 r \pi u}{h}\right) w_{u}^{j} \mathrm{~d} u\right)\right. \\
& \left.-b_{r}^{i} \int_{0}^{h} \frac{2 r \pi}{h} \cos \left(\frac{2 r \pi u}{h}\right) w_{u}^{j} \mathrm{~d} u\right)
\end{aligned}
$$

The expression $a_{0}^{j}=\frac{2}{h} \int_{0}^{h} w_{u}^{j} \mathrm{~d} u-w_{h}^{j}$ and the definition of $a_{r}^{j}, b_{r}^{j}, r \in \mathbb{N}$ as well as in the last step $a_{0}^{j}=-2 \sum_{r=1}^{\infty} a_{r}^{j}, j \in \mathcal{J}_{K}$ yield

$$
\begin{aligned}
I_{(i, j)}^{Q}(h) & =\frac{1}{2} w_{h}^{i} w_{h}^{j}-\frac{1}{2}\left(a_{0}^{j} w_{h}^{i}-a_{0}^{i} w_{h}^{j}\right) \\
& +\sum_{r=1}^{\infty}\left(a_{r}^{i}\left(w_{h}^{j}+r \pi\left(b_{r}^{j}-\frac{w_{h}^{j}}{r \pi}\right)\right)-r \pi b_{r}^{i} a_{r}^{j}\right) \\
& =\frac{1}{2} w_{h}^{i} w_{h}^{j}-\frac{1}{2}\left(a_{0}^{j} w_{h}^{i}-a_{0}^{i} w_{h}^{j}\right)+\pi \sum_{r=1}^{\infty} r\left(a_{r}^{i} b_{r}^{j}-b_{r}^{i} a_{r}^{j}\right) \\
& =\frac{1}{2} \Delta w_{h}^{i} \Delta w_{h}^{j}+\pi \sum_{r=1}^{\infty} r\left(a_{r}^{i}\left(b_{r}^{j}-\frac{1}{\pi r} \Delta w_{h}^{j}\right)-\left(b_{r}^{i}-\frac{1}{\pi r} \Delta w_{h}^{i}\right) a_{r}^{j}\right)
\end{aligned}
$$

for all $i, j \in \mathcal{J}_{K}, i \neq j$, and $h>0$. Expression (12) involves some scaled Lévy stochastic area integrals which are defined as

$$
A_{(i, j)}^{Q}(h):=\pi \sum_{r=1}^{\infty} r\left(a_{r}^{i}\left(b_{r}^{j}-\frac{1}{\pi r} \Delta w_{h}^{j}\right)-\left(b_{r}^{i}-\frac{1}{\pi r} \Delta w_{h}^{i}\right) a_{r}^{j}\right)
$$

for all $i, j \in \mathcal{J}_{K}, i \neq j, h>0$. We approximate these terms instead of the iterated stochastic integrals, as proposed in [4] and [9]. Due to the relations

$$
\begin{aligned}
& I_{(i, j)}^{Q}(h)=\frac{\Delta w_{h}^{i} \Delta w_{h}^{j}-h \eta_{i} \mathbb{1}_{i=j}}{2}+A_{(i, j)}^{Q}(h) \\
& A_{(j, i)}^{Q}(h)=-A_{(i, j)}^{Q}(h) \\
& A_{(i, i)}^{Q}(h)=0
\end{aligned}
$$


$P$-a.s. for all $i, j \in \mathcal{J}_{K}, h>0$, see [9], it is sufficient to simulate $A_{(i, j)}^{Q}(h)$ for $i, j \in \mathcal{J}_{K}$ with $i<j$. By the distributional properties of $a_{r}^{i}$ and $b_{r}^{i}$ for $r \in \mathbb{N}_{0}$, $i \in \mathcal{J}_{K}$, we write

$$
A_{(i, j)}^{Q}(h)=\frac{h}{2 \pi} \sum_{r=1}^{\infty} \frac{1}{r}\left(U_{r i}^{Q}\left(Z_{r j}^{Q}-\sqrt{\frac{2}{h}} \Delta w_{h}^{j}\right)-U_{r j}^{Q}\left(Z_{r i}^{Q}-\sqrt{\frac{2}{h}} \Delta w_{h}^{i}\right)\right)
$$

for all $i, j \in \mathcal{J}_{K}, i \neq j, h>0$ and $A^{Q}(h)=\left(A_{(i, j)}^{Q}(h)\right)_{1 \leq i, j \leq K}$ in order to relate to the derivation in [9]. This representation entails the random variables $U_{r i}^{Q} \sim N\left(0, \eta_{i}\right)$, $Z_{r i}^{Q} \sim N\left(0, \eta_{i}\right)$, and $\Delta w_{h}^{i} \sim N\left(0, \eta_{i} h\right)$ that are all independent for $i \in \mathcal{J}_{K}, r \in \mathbb{N}$. As described above, we only need to approximate $A_{(i, j)}^{Q}(h), h>0$ for $i, j \in \mathcal{J}_{K}$ with $i<j$, that is, we want to simulate

$$
\begin{aligned}
\tilde{A}^{Q}(h)= & \left(A_{1,2}^{Q}(h), \ldots, A_{1, K}^{Q}(h), A_{2,3}^{Q}(h), \ldots, A_{2, K}^{Q}(h), \ldots, A_{l, l+1}^{Q}(h), \ldots,\right. \\
& \left.A_{l, K}^{Q}(h), \ldots, A_{K-1, K}^{Q}(h)\right) .
\end{aligned}
$$

Therefore, we write

$$
\operatorname{vec}\left(A^{Q}(h)^{T}\right)=\left(A_{1,1}^{Q}(h), \ldots, A_{1, K}^{Q}(h), \ldots, A_{K, 1}^{Q}(h), \ldots, A_{K, K}^{Q}(h)\right)^{T}
$$

and introduce the selection matrix

$$
H_{K}=\left(\begin{array}{ccc}
0_{K-1 \times 1} & I_{K-1} & 0_{K-1 \times K(K-1)} \\
0_{K-2 \times K+2} & I_{K-2} & 0_{K-2 \times K(K-2)} \\
\vdots & \vdots & \vdots \\
0_{K-l \times(l-1) K+l} & I_{K-l} & 0_{K-l \times K(K-l)} \\
\vdots & \vdots & \vdots \\
0_{1 \times(K-2) K+K-1} & 1 & 0_{1 \times K}
\end{array}\right)
$$

which defines the integrals that have to be computed, compare to [9]. Note that $I_{K}$ represents the identity matrix of size $K \times K$. Further, we define the matrix

$$
Q_{K}:=\operatorname{diag}\left(\eta_{1}, \ldots, \eta_{K}\right)
$$

This allows to express the vector $\tilde{A}^{Q}(h)$ as

$$
\begin{aligned}
\tilde{A}^{Q}(h) & =H_{K} \operatorname{vec}\left(A^{Q}(h)^{T}\right) \\
& =\frac{h}{2 \pi} \sum_{r=1}^{\infty} \frac{1}{r} H_{K}\left(U_{r}^{Q} \otimes\left(Z_{r}^{Q}-\sqrt{\frac{2}{h}} \Delta w_{h}^{Q}\right)-\left(Z_{r}^{Q}-\sqrt{\frac{2}{h}} \Delta w_{h}^{Q}\right) \otimes U_{r}^{Q}\right)
\end{aligned}
$$


with $\Delta w_{h}^{Q}=\left(\Delta w_{h}^{1}, \ldots, \Delta w_{h}^{K}\right)^{T}$ and the random vectors $U_{r}^{Q}=\left(U_{r 1}^{Q}, \ldots, U_{r K}^{Q}\right)^{T}$ and $Z_{r}^{Q}=\left(Z_{r 1}^{Q}, \ldots, Z_{r K}^{Q}\right)^{T}$ that are independent and identically $N\left(0_{K}, Q_{K}\right)$ distributed for all $r \in \mathbb{N}$. As expression (18) contains an infinite sum, we need to truncate it in order to compute this vector. For some $D \in \mathbb{N}$, this approximation is denoted as

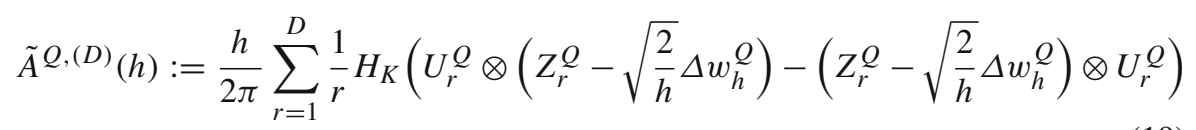

and we specify the remainder

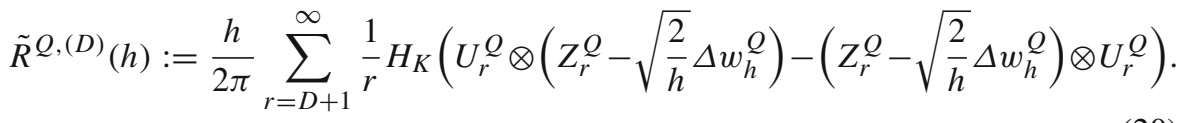

Let $A^{I}(h)=Q_{K}^{-1 / 2} A^{Q}(h) Q_{K}^{-1 / 2}$ denote the matrix containing the standard Lévy stochastic area integrals that correspond to the case that $Q_{K}=I_{K}$, i.e., $\eta_{j}=1$ for all $j \in \mathcal{J}_{K}$. Therewith, we obtain the relationship

$$
\begin{aligned}
\tilde{A}^{Q}(h) & =H_{K} \operatorname{vec}\left(A^{Q}(h)^{T}\right) \\
& =H_{K}\left(Q_{K}^{1 / 2} \otimes Q_{K}^{1 / 2}\right) \operatorname{vec}\left(A^{I}(h)^{T}\right) \\
& =H_{K}\left(Q_{K}^{1 / 2} \otimes Q_{K}^{1 / 2}\right) H_{K}^{T} H_{K} \operatorname{vec}\left(A^{I}(h)^{T}\right) \\
& =H_{K}\left(Q_{K}^{1 / 2} \otimes Q_{K}^{1 / 2}\right) H_{K}^{T} \tilde{A}^{I}(h),
\end{aligned}
$$

where $\tilde{A}^{I}(h):=H_{K} \operatorname{vec}\left(A^{I}(h)^{T}\right)$ and where we employed

$$
H_{K}^{T} H_{K}=\operatorname{diag}\left(0, \mathbf{1}_{K-1}^{T}, 0,0, \mathbf{1}_{K-2}^{T}, \ldots, \mathbf{0}_{l}^{T}, \mathbf{1}_{K-l}^{T}, \ldots, \mathbf{0}_{K-1}^{T}, 1, \mathbf{0}_{K}^{T}\right) \in \mathbb{R}^{K^{2} \times K^{2}}
$$

and the fact that we are interested in indices $i, j \in \mathcal{J}_{K}$ with $i<j$ only. We denote

$$
\tilde{Q}_{K}:=H_{K}\left(Q_{K}^{1 / 2} \otimes Q_{K}^{1 / 2}\right) H_{K}^{T},
$$

which is of size $L \times L$ with $L=\frac{K(K-1)}{2}$, such that the vector of interest is given by

$$
\tilde{A}^{Q}(h)=\tilde{Q}_{K} \tilde{A}^{I}(h) .
$$

Now, we can represent the approximation $\tilde{A}^{Q,(D)}(h)$ of $\tilde{A}^{Q}(h)$ as

$$
\tilde{A}^{Q,(D)}(h)=\tilde{Q}_{K} \tilde{A}^{I,(D)}(h)
$$

and the vector of truncation errors by $\tilde{R}^{Q,(D)}(h)=\tilde{Q}_{K} \tilde{R}^{I,(D)}(h)$ where $\tilde{A}^{I,(D)}(h)$ and $\tilde{R}^{I,(D)}(h)$ denote, in analogy to (19) and (20), the truncated part of $\tilde{A}^{I}(h)$ and its truncation error, respectively. Note that $\tilde{A}^{I}(h)$ and especially $\tilde{A}^{I,(D)}(h)$ correspond to 
the case where $\eta_{j}=1$ for all $j \in \mathcal{J}_{K}$, i.e., $Q_{K}=I_{K}$ in (18) and (19), respectively. This also relates to the setting in [4] if $\mathcal{J}$ is finite.

We summarize the representation above to formulate Algorithm 1 for some $h>0$, $t, t+h \in[0, T]$, and $D, K \in \mathbb{N}$ :

1. For $j \in \mathcal{J}_{K}$, simulate the Fourier coefficients $\Delta w_{h}^{j}=\left\langle W_{t+h}-W_{t}, \tilde{e}_{j}\right\rangle_{U}$ of the increment $W_{t+h}-W_{t}$ with $\Delta w_{h}^{Q}=\left(\Delta w_{h}^{1}, \ldots, \Delta w_{h}^{K}\right)^{T}$ as

$$
\Delta w_{h}^{Q}=\sqrt{h} Q_{K}^{1 / 2} V
$$

where $V \sim N\left(0_{K}, I_{K}\right)$.

2. Approximate $\tilde{A}^{Q}(h)$ as

$$
\begin{aligned}
\tilde{A}^{Q,(D)}(h)= & H_{K}\left(Q_{K}^{1 / 2} \otimes Q_{K}^{1 / 2}\right) H_{K}^{T} \\
& \times \frac{h}{2 \pi} \sum_{r=1}^{D} \frac{1}{r} H_{K}\left(U_{r} \otimes\left(Z_{r}-\sqrt{2} V\right)-\left(Z_{r}-\sqrt{2} V\right) \otimes U_{r}\right),
\end{aligned}
$$

where $U_{r}, Z_{r} \sim N\left(0_{K}, I_{K}\right)$ are independent.

2. Compute the approximation $\operatorname{vec}\left(\left(\bar{I}^{Q,(D)}(h)\right)^{T}\right)$ of $\operatorname{vec}\left(\left(I^{Q}(h)^{T}\right)\right.$ as

$$
\operatorname{vec}\left(\left(\bar{I}^{Q,(D)}(h)\right)^{T}\right)=\frac{\Delta w_{h}^{Q} \otimes \Delta w_{h}^{Q}-\operatorname{vec}\left(h Q_{K}\right)}{2}+\left(I_{K^{2}}-S_{K}\right) H_{K}^{T} \tilde{A}^{Q,(D)}(h)
$$

with $S_{K}:=\sum_{i=1}^{K} \mathbf{e}_{i}^{T} \otimes\left(I_{K} \otimes \mathbf{e}_{i}\right)$, where $\mathbf{e}_{i}$ denotes the $i$-th unit vector.

We obtain the following error estimate for this approximation method; the meansquare error converges with order $1 / 2$ in $D$ while the convergence in $K$ is determined by the operator $Q$. The first term results from the approximation of the $Q$-Wiener process by $\left(W_{t}^{K}\right)_{t \geq 0}$, whereas the second term is due to the approximation of the iterated integral with respect to this truncated process by Algorithm 1.

Theorem 1 (Convergence result for Algorithm 1) Assume that the trace class operator $Q \in L(U)$ is nonnegative and symmetric with eigenvalues $\left\{\eta_{j}: j \in \mathcal{J}\right\}$. Further, let $\Phi \in L(U, H)_{U_{0}}$ with $\left\|\Phi Q^{-\alpha}\right\|_{L_{H S}\left(U_{0}, H\right)}<C$ for some $C>0$, let $\Psi \in L\left(H, L\left(Q^{-\alpha} U, H\right)_{U_{0}}\right)$ for some $\alpha \in(0, \infty)$, i.e., (A1) and (A2) are fulfilled, and let $\left(W_{t}\right)_{t \geq 0}$ be a $Q$-Wiener process. Then, it holds

$$
\begin{aligned}
& \left(\mathrm{E}\left[\left\|\int_{t}^{t+h} \Psi\left(\int_{t}^{s} \Phi \mathrm{d} W_{r}\right) \mathrm{d} W_{s}-\sum_{i, j \in \mathcal{J}_{K}} \bar{I}_{(i, j)}^{Q,(D)}(h) \Psi\left(\Phi \tilde{e}_{i}, \tilde{e}_{j}\right)\right\|_{H}^{2}\right]\right)^{\frac{1}{2}} \\
& \quad \leq C\left(h\left(\sup _{j \in \mathcal{J} \backslash \mathcal{J}_{K}} \eta_{j}\right)^{\alpha}+\operatorname{tr} Q \frac{h}{\pi \sqrt{D}}\right)
\end{aligned}
$$

for some $C>0$ and all $h>0, t, t+h \in[0, T], D, K \in \mathbb{N}$, and $\mathcal{J}_{K} \subset \mathcal{J}$ with $\left|\mathcal{J}_{K}\right|=K$.

Proof For a proof, we refer to Sect. 3. 
Note that in the convergence analysis of numerical schemes for SPDEs, we compare the approximation of the iterated stochastic integrals to integrals with respect to $\left(W_{t}^{K}\right)_{t \geq 0}$, $K \in \mathbb{N}$, see the proofs in [2] and [6], for example. In this case, the analysis involves the error estimate stated in Corollary 1 below. We want to emphasize that this estimate is independent of the integer $K$.

Corollary 1 Assume that $Q$ is a nonnegative and symmetric trace class operator and $\left(W_{t}\right)_{t \geq 0}$ is a $Q$-Wiener process. Furthermore, let $\Phi \in L(U, H)_{U_{0}}$ and $\Psi \in$ $L\left(H, L(U, H)_{U_{0}}\right)$, i.e., assumptions $(B 1)$ and (B2) are fulfilled. Then, it holds

$$
\begin{aligned}
& \left(\mathrm{E}\left[\left\|\int_{t}^{t+h} \Psi\left(\int_{t}^{s} \Phi \mathrm{d} W_{r}^{K}\right) \mathrm{d} W_{s}^{K}-\sum_{i, j \in \mathcal{J}_{K}} \bar{I}_{(i, j)}^{Q,(D)}(h) \Psi\left(\Phi \tilde{e}_{i}, \tilde{e}_{j}\right)\right\|_{H}^{2}\right]\right)^{\frac{1}{2}} \\
& \quad \leq C \operatorname{tr} Q \frac{h}{\pi \sqrt{D}}
\end{aligned}
$$

for some $C>0$ and all $h>0, t, t+h \in[0, T], D, K \in \mathbb{N}$, and $\mathcal{J}_{K} \subset \mathcal{J}$ with $\left|\mathcal{J}_{K}\right|=K$.

Proof If we set $\eta_{i}=0$ for all $i \in \mathcal{J} \backslash \mathcal{J}_{K}$, the result follows directly from Theorem 1.

Next, we outline an alternative algorithm to approximate integrals of type (7). In contrast to the method presented above, the vector of tail sum $\tilde{R}^{Q,(D)}(h)$ is approximated and included in the computation.

\subsection{Algorithm 2}

The following derivation is based on the scheme developed by Wiktorsson [9] for SDEs. In the finite dimensional setting, the error estimate (4) depends on the number of Brownian motions $K$ additionally to the time step size $h$. This suggests that the computational cost involved in the simulation of the stochastic double integrals is much larger in the setting of SPDEs as the number of independent Brownian motions is, in general, not finite, see also expression (8). The eigenvalues of the $Q$-Wiener process are, however, not incorporated in the error estimate (4). For example, if we assume $\eta_{j}=\mathcal{O}\left(j^{-\rho_{Q}}\right), j \in \mathcal{J} \subset \mathbb{N}$, we obtain for $\rho_{Q} \in(1,3)$ an improved error estimate which depends on the rate of decay of the eigenvalues instead of some fixed exponent of $K$. This results from the fact that we integrate with respect to a $Q$-Wiener process in our setting, where $Q$ is a nonnegative, symmetric trace class operator. For $\rho_{Q} \geq 3$, we can show that the exponent of $K$ is bounded by 3 .

As before, we truncate the series (18) at some integer $D \in \mathbb{N}$ and obtain the approximation $\tilde{A}^{Q,(D)}(h)$ in (19). The vector of tail sum $\tilde{R}^{Q,(D)}(h)$ in (20), however, is not discarded but approximated by a multivariate normally distributed random vector instead, as described in [9] for $Q_{K}=I_{K}$ and $|\mathcal{J}|=K$. First, we determine the 
distribution of the tail sum; for $r \in \mathbb{N}$, we compute the covariance matrix of

$$
\begin{aligned}
V_{r}^{Q} & :=U_{r}^{Q} \otimes\left(Z_{r}^{Q}-\sqrt{\frac{2}{h}} \Delta w_{h}^{Q}\right)-\left(Z_{r}^{Q}-\sqrt{\left.\frac{2}{h} \Delta w_{h}^{Q}\right) \otimes U_{r}^{Q}}\right. \\
& =\left(S_{K}-I_{K^{2}}\right)\left(\left(Z_{r}^{Q}-\sqrt{\frac{2}{h}} \Delta w_{h}^{Q}\right) \otimes U_{r}^{Q}\right)
\end{aligned}
$$

conditional on $Z_{r}^{Q}$ and $\Delta w_{h}^{Q}$ with $S_{K}=\sum_{i=1}^{K} \mathbf{e}_{i}^{T} \otimes\left(I_{K} \otimes \mathbf{e}_{i}\right)$, where $\mathbf{e}_{i}$ denotes the $i$-th unit vector. Note that $S_{K}(x \otimes y)=y \otimes x$ for any vectors $x, y \in \mathbb{R}^{K}$ [9]. Considering that $U_{r}^{Q}$ is a standard normally distributed vector, the covariance matrix results in

$$
\begin{aligned}
\Sigma^{Q}\left(V_{r}^{Q}\right)_{\mid Z_{r}^{Q}, \Delta w_{h}^{Q}}= & \mathrm{E}\left[V_{r}^{Q} V_{r}^{Q^{T}} \mid Z_{r}^{Q}, \Delta w_{h}^{Q}\right] \\
& -\mathrm{E}\left[V_{r}^{Q} \mid Z_{r}^{Q}, \Delta w_{h}^{Q}\right] \mathrm{E}\left[V_{r}^{Q} \mid Z_{r}^{Q}, \Delta w_{h}^{Q}\right]^{T} \\
= & \left(S_{K}-I_{K^{2}}\right)\left(\left(Z_{r}^{Q}-\sqrt{\frac{2}{h}} \Delta w_{h}^{Q}\right)\left(Z_{r}^{Q}-\sqrt{\frac{2}{h}} \Delta w_{h}^{Q}\right)^{T} \otimes Q_{K}\right) \\
& \times\left(S_{K}-I_{K^{2}}\right) .
\end{aligned}
$$

This expression can be reformulated without using the operator $S_{K}$ by taking into account that

$$
\begin{aligned}
\mathrm{E} & {\left[U_{r}^{Q}\left(Z_{r}^{Q}-\sqrt{\frac{2}{h}} \Delta w_{h}^{Q}\right)^{T} \otimes\left(Z_{r}^{Q}-\sqrt{\frac{2}{h}} \Delta w_{h}^{Q}\right) U_{r}^{Q^{T}} \mid Z_{r}^{Q}, \Delta w_{h}^{Q}\right] } \\
= & \left(I_{K} \otimes \operatorname{diag}\left(Z_{r}^{Q}-\sqrt{\frac{2}{h}} \Delta w_{h}^{Q}\right)\right)\left(\mathbf{1}_{K}^{T} \otimes\left(Q_{K} \otimes \mathbf{1}_{K}\right)\right) \\
& \times\left(\operatorname{diag}\left(Z_{r}^{Q}-\sqrt{\frac{2}{h}} \Delta w_{h}^{Q}\right) \otimes I_{K}\right) \\
= & \left(Q_{K}^{1 / 2} \otimes \operatorname{diag}\left(Z_{r}^{Q}-\sqrt{\frac{2}{h}} \Delta w_{h}^{Q}\right)\right)\left(\left(Z_{r}^{Q}-\sqrt{\frac{2}{h}} \Delta w_{h}^{Q}\right)^{T} \otimes\left(Q_{K}^{1 / 2} \otimes \mathbf{1}_{K}\right)\right)
\end{aligned}
$$

as

$$
\begin{aligned}
& \Sigma^{Q}\left(V_{r}^{Q}\right)_{\mid Z_{r}^{Q}, \Delta w_{h}^{Q}} \\
& =Q_{K} \otimes\left(Z_{r}^{Q}-\sqrt{\frac{2}{h}} \Delta w_{h}^{Q}\right)\left(Z_{r}^{Q}-\sqrt{\frac{2}{h}} \Delta w_{h}^{Q}\right)^{T} \\
& +\left(Z_{r}^{Q}-\sqrt{\frac{2}{h}} \Delta w_{h}^{Q}\right)\left(Z_{r}^{Q}-\sqrt{\frac{2}{h}} \Delta w_{h}^{Q}\right)^{T} \otimes Q_{K} \\
& -\left(Q_{K}^{1 / 2} \otimes \operatorname{diag}\left(Z_{r}^{Q}-\sqrt{\frac{2}{h}} \Delta w_{h}^{Q}\right)\right)\left(\left(Z_{r}^{Q}-\sqrt{\frac{2}{h}} \Delta w_{h}^{Q}\right)^{T} \otimes\left(Q_{K}^{1 / 2} \otimes \mathbf{1}_{K}\right)\right)
\end{aligned}
$$


$-\left(\left(Z_{r}^{Q}-\sqrt{\frac{2}{h}} \Delta w_{h}^{Q}\right) \otimes\left(Q_{K}^{1 / 2} \otimes \mathbf{1}_{K}^{T}\right)\right)\left(Q_{K}^{1 / 2} \otimes \operatorname{diag}\left(Z_{r}^{Q}-\sqrt{\frac{2}{h}} \Delta w_{h}^{Q}\right)\right)$.

Analogously to [9], by taking the expectation, we define

$$
\begin{aligned}
\Sigma_{\infty}^{Q}:= & \mathrm{E}\left[H_{K} \Sigma^{Q}\left(V_{1}^{Q}\right)_{\mid Z_{1}^{Q}, \Delta w_{h}^{Q}} H_{K}^{T} \mid \Delta w_{h}^{Q}\right] \\
= & 2 H_{K}\left(Q_{K} \otimes Q_{K}\right) H_{K}^{T} \\
& +\frac{2}{h} H_{K}\left(I_{K^{2}}-S_{K}\right)\left(Q_{K} \otimes\left(\Delta w_{h}^{Q} \Delta w_{h}^{Q^{T}}\right)\right)\left(I_{K^{2}}-S_{K}\right) H_{K}^{T}
\end{aligned}
$$

Taking into consideration that

$$
\begin{aligned}
& \mathrm{E}\left[\left(I_{K} \otimes \operatorname{diag}\left(Z_{r}^{Q}-\sqrt{\frac{2}{h}} \Delta w_{h}^{Q}\right)\right)\left(\mathbf{1}_{K}^{T} \otimes\left(Q_{K} \otimes \mathbf{1}_{K}\right)\right)\right. \\
& \quad \times\left(\operatorname { d i a g } \left(Z_{r}^{Q}-\sqrt{\left.\left.\left.\frac{2}{h} \Delta w_{h}^{Q}\right) \otimes I_{K}\right) \mid \Delta w_{h}^{Q}\right]}\right.\right. \\
& =\frac{2}{h}\left(I_{K} \otimes \operatorname{diag}\left(\Delta w_{h}^{Q}\right)\right)\left(\mathbf{1}_{K}^{T} \otimes\left(Q_{K} \otimes \mathbf{1}_{K}\right)\right)\left(\operatorname{diag}\left(\Delta w_{h}^{Q}\right) \otimes I_{K}\right) \\
& \quad+\sum_{i=1}^{K}\left(Q_{K}^{1 / 2} \mathrm{e}_{i}\right)^{T} \otimes\left(I_{K} \otimes Q_{K}^{1 / 2} \mathrm{e}_{i}\right)
\end{aligned}
$$

and that $H_{K}\left(\sum_{i=1}^{K}\left(Q_{K}^{1 / 2} \mathrm{e}_{i}\right)^{T} \otimes\left(I_{K} \otimes Q_{K}^{1 / 2} \mathrm{e}_{i}\right)\right) H_{K}^{T}=0$, it follows that expression (23) can be rewritten as

$$
\begin{aligned}
\Sigma_{\infty}^{Q}= & 2 H_{K}\left(Q_{K} \otimes Q_{K}\right) H_{K}^{T}+\frac{2}{h} H_{K}\left(Q_{K} \otimes \Delta w_{h}^{Q} \Delta w_{h}^{Q^{T}}+\Delta w_{h}^{Q} \Delta w_{h}^{Q^{T}} \otimes Q_{K}\right. \\
& -\left(Q_{K}^{1 / 2} \otimes \operatorname{diag}\left(\Delta w_{h}^{Q}\right)\right)\left(\Delta w_{h}^{Q^{T}} \otimes\left(Q_{K}^{1 / 2} \otimes \mathbf{1}_{K}\right)\right) \\
& \left.-\left(\Delta w_{h}^{Q} \otimes\left(Q_{K}^{1 / 2} \otimes \mathbf{1}_{K}^{T}\right)\right)\left(Q_{K}^{1 / 2} \otimes \operatorname{diag}\left(\Delta w_{h}^{Q}\right)\right)\right) H_{K}^{T}
\end{aligned}
$$

The definitions above imply that, given $Z^{Q}=\left(Z_{r}^{Q}\right)_{r \in \mathbb{N}}$ and $\Delta w_{h}^{Q}$, the vector of tail sum $\tilde{R}^{Q,(D)}(h)$ is conditionally Gaussian distributed with the following parameters

$$
\tilde{R}^{Q,(D)}(h)_{\mid Z Q, \Delta w_{h}^{Q}} \sim N\left(0_{L},\left(\frac{h}{2 \pi}\right)^{2} \sum_{r=D+1}^{\infty} \frac{1}{r^{2}} H_{K} \Sigma^{Q}\left(V_{r}^{Q}\right)_{\mid Z_{r}^{Q}, \Delta w_{h}^{Q}} H_{K}^{T}\right)
$$

for $D \in \mathbb{N}$. Hence, given $Z^{Q}$ and $\Delta w_{h}^{Q}$, we can approximate the tail sum by simulating a conditionally standard Gaussian random vector $\Upsilon_{\mid Z{ }^{Q}, \Delta w_{h}^{Q}}^{Q,(D)} \sim N\left(0_{L}, I_{L}\right)$ 
defined as

$$
\Upsilon^{Q,(D)}=\frac{2 \pi}{h}\left(\sum_{r=D+1}^{\infty} \frac{1}{r^{2}} H_{K} \Sigma^{Q}\left(V_{r}^{Q}\right)_{\mid Z_{r}^{Q}, \Delta w_{h}^{Q} H_{K}^{T}}\right)^{-\frac{1}{2}} \tilde{R}^{Q,(D)}(h)
$$

and, therewith, obtain the vector of tail sum

$$
\tilde{R}^{Q,(D)}(h)=\frac{h}{2 \pi}\left(\sum_{r=D+1}^{\infty} \frac{1}{r^{2}} H_{K} \Sigma^{Q}\left(V_{r}^{Q}\right)_{\mid Z_{r}^{Q}, \Delta w_{h}^{Q}} H_{K}^{T}\right)^{\frac{1}{2}} \Upsilon^{Q,(D)} .
$$

It remains to examine how the covariance matrix evolves as $D \rightarrow \infty$. For $D \in \mathbb{N}$, we define the matrix

$$
\Sigma^{Q,(D)}:=\left(\sum_{r=D+1}^{\infty} \frac{1}{r^{2}}\right)^{-1} \sum_{r=D+1}^{\infty} \frac{H_{K} \Sigma^{Q}\left(V_{r}^{Q}\right)_{\mid Z_{r}^{Q}, \Delta w_{h}^{Q}} H_{K}^{T}}{r^{2}} .
$$

By the proof of Theorem 3 below, we get convergence in the following sense

$$
\lim _{D \rightarrow \infty} \mathrm{E}\left[\left\|\Sigma^{Q,(D)}-\Sigma_{\infty}^{Q}\right\|_{F}^{2}\right]=0
$$

where $\|\cdot\|_{F}$ denotes the Frobenius norm. Thus, it follows

$$
\frac{2 \pi}{h}\left(\sum_{r=D+1}^{\infty} \frac{1}{r^{2}}\right)^{-\frac{1}{2}} \tilde{R}^{Q,(D)}(h) \stackrel{d}{\longrightarrow} \xi \sim N\left(0_{L}, \Sigma_{\infty}^{Q}\right)
$$

as $D \rightarrow \infty$, see also [9].

Combining the above, we obtain an algorithm very similar to the one in [9], where steps 1,2, and 4 equal Algorithm 1. Additionally, we approximate the vector of tail sum in step 3. For some $h>0, t, t+h \in[0, T]$, and $D, K \in \mathbb{N}$, Algorithm 2 is defined as follows:

1. For $j \in \mathcal{J}_{K}$, simulate the Fourier coefficients $\Delta w_{h}^{j}=\left\langle W_{t+h}-W_{t}, \tilde{e}_{j}\right\rangle_{U}$ of the increment $W_{t+h}-W_{t}$ with $\Delta w_{h}^{Q}=\left(\Delta w_{h}^{1}, \ldots, \Delta w_{h}^{K}\right)^{T}$ as

$$
\Delta w_{h}^{Q}=\sqrt{h} Q_{K}^{1 / 2} V
$$

where $V \sim N\left(0_{K}, I_{K}\right)$.

2. Approximate $\tilde{A}^{Q}(h)$ as

$$
\begin{aligned}
\tilde{A}^{Q,(D)}(h)= & H_{K}\left(Q_{K}^{1 / 2} \otimes Q_{K}^{1 / 2}\right) H_{K}^{T} \\
& \times \frac{h}{2 \pi} \sum_{r=1}^{D} \frac{1}{r} H_{K}\left(U_{r} \otimes\left(Z_{r}-\sqrt{2} V\right)-\left(Z_{r}-\sqrt{2} V\right) \otimes U_{r}\right),
\end{aligned}
$$


where $U_{r}, Z_{r} \sim N\left(0_{K}, I_{K}\right)$ are independent.

3. Simulate $\Upsilon^{Q,(D)} \sim N\left(0_{L}, I_{L}\right)$ and compute

$$
\hat{A}^{Q,(D)}(h)=\tilde{A}^{Q,(D)}(h)+\frac{h}{2 \pi}\left(\sum_{r=D+1}^{\infty} \frac{1}{r^{2}}\right)^{\frac{1}{2}} \sqrt{\Sigma_{\infty}^{Q}} \Upsilon^{Q,(D)} .
$$

4. Compute the approximation $\operatorname{vec}\left(\left(\hat{I}^{Q,(D)}(h)\right)^{T}\right)$ of $\operatorname{vec}\left(\left(I^{Q}(h)^{T}\right)\right.$ as

$$
\begin{aligned}
& \operatorname{vec}\left(\left(\hat{I}^{Q,(D)}(h)\right)^{T}\right)=\frac{\Delta w_{h}^{Q} \otimes \Delta w_{h}^{Q}-\operatorname{vec}\left(h Q_{K}\right)}{2}+\left(I_{K^{2}}-S_{K}\right) H_{K}^{T} \hat{A}^{Q,(D)}(h) \\
& \text { with } S_{K}=\sum_{i=1}^{K} \mathbf{e}_{i}^{T} \otimes\left(I_{K} \otimes \mathbf{e}_{i}\right) .
\end{aligned}
$$

Note that the matrix $\sqrt{\Sigma_{\infty}^{Q}}$ in step 3 may be calculated by a Cholesky decomposition. However, the square root of $\Sigma_{\infty}^{Q}$ can be obtained in closed form and does not have to be computed numerically.

Theorem 2 Let $\Sigma_{\infty}^{Q}$ be defined as in (23) with $\Delta w_{h}^{Q}=\sqrt{h} Q_{K}^{1 / 2} V$ and let $\Sigma_{\infty}^{I}$ be defined by (23) with $Q_{K}=I_{K}$. Then, it holds

$$
\sqrt{\Sigma_{\infty}^{Q}}=\tilde{Q}_{K} \frac{\Sigma_{\infty}^{I}+2 \sqrt{1+V^{T} V} I_{L}}{\sqrt{2}\left(1+\sqrt{1+V^{T} V}\right)}
$$

Proof For a proof, we refer to Sect. 3.

Now, we analyze the error resulting from Algorithm 2. In the following theorem, the first term is the same as in the error estimate of Algorithm 1, see Theorem 1. Due to the second term, the approximation converges with order 1 in $D$, which is twice the order that Algorithm 1 attains. However, this expression is dependent on $K$ as well. Note that $\max _{j \in \mathcal{J}_{K}} \eta_{j} \leq \max _{j \in \mathcal{J}} \eta_{j} \leq \operatorname{tr} Q<\infty$. Below, we state an alternative estimate-there, the exponent of $K$ is not fixed but dependent on the eigenvalues $\eta_{j}$, $j \in \mathcal{J}_{K}$, see Theorem 4. The algorithm that is superior can only be determined in dependence on the operator $Q$.

Theorem 3 (Convergence result for Algorithm 2) Assume that the trace class operator $Q$ is nonnegative and symmetric and $\left(W_{t}\right)_{t \geq 0}$ is a $Q$-Wiener process. Further, let $\Phi \in L(U, H)_{U_{0}}$ with $\left\|\Phi Q^{-\alpha}\right\|_{L_{H S}\left(U_{0}, H\right)}<C$ for some $C>0$, let $\Psi \in$ $L\left(H, L\left(Q^{-\alpha} U, H\right)_{U_{0}}\right)$ for some $\alpha \in(0, \infty)$, i.e., assumptions $(A 1)$ and $(A 2)$ are fulfilled. Then, it holds

$$
\begin{aligned}
& \left(\mathrm{E}\left[\left\|\int_{t}^{t+h} \Psi\left(\int_{t}^{s} \Phi \mathrm{d} W_{r}\right) \mathrm{d} W_{s}-\sum_{i, j \in \mathcal{J}_{K}} \hat{I}_{(i, j)}^{Q,(D)}(h) \Psi\left(\Phi \tilde{e}_{i}, \tilde{e}_{j}\right)\right\|_{H}^{2}\right]\right)^{\frac{1}{2}} \\
& \quad \leq C\left(h\left(\sup _{j \in \mathcal{J} \backslash \mathcal{J}_{K}} \eta_{j}\right)^{\alpha}+\max _{j \in \mathcal{J}_{K}} \eta_{j} \sqrt{K^{2}(K-1)} \frac{h}{D}\right)
\end{aligned}
$$


for some $C>0$ and all $h>0, t, t+h \in[0, T], D, K \in \mathbb{N}$, and $\mathcal{J}_{K} \subset \mathcal{J}$ with $\left|\mathcal{J}_{K}\right|=K$.

Proof For a proof, we refer to Sect. 3.

For completeness, we state the following error estimate. Again, this is the estimate that we consider when incorporating the approximation of the iterated integrals into a numerical scheme; see also the notes on Corollary 1.

Corollary 2 Assume that $Q$ is a nonnegative and symmetric trace class operator and $\left(W_{t}\right)_{t \geq 0}$ is a $Q$-Wiener process. Furthermore, let $\Phi \in L(U, H)_{U_{0}}, \Psi \in$ $L\left(H, L(U, H)_{U_{0}}\right)$, i.e., conditions (B1) and (B2) are fulfilled. Then, it holds

$$
\begin{aligned}
& \left(\mathrm{E}\left[\left\|\int_{t}^{t+h} \Psi\left(\int_{t}^{s} \Phi \mathrm{d} W_{r}^{K}\right) \mathrm{d} W_{s}^{K}-\sum_{i, j \in \mathcal{J}_{K}} \hat{I}_{(i, j)}^{Q,(D)}(h) \Psi\left(\Phi \tilde{e}_{i}, \tilde{e}_{j}\right)\right\|_{H}^{2}\right]\right)^{\frac{1}{2}} \\
& \quad \leq C \max _{j \in \mathcal{J}_{K}} \eta_{j} \sqrt{K^{2}(K-1)} \frac{h}{D}
\end{aligned}
$$

for some $C>0$ and all $h>0, t, t+h \in[0, T], D, K \in \mathbb{N}$, and $\mathcal{J}_{K} \subset \mathcal{J}$ with $\left|\mathcal{J}_{K}\right|=K$.

Proof The proof of this corollary is detailed in the proof of Theorem 3.

If we assume, e.g., $\eta_{j} \leq C j^{-\rho_{Q}}$ for $C>0, \rho_{Q}>1$, and all $j \in \mathcal{J} \subset \mathbb{N}$, we can improve the result in Theorem 3 in the case $\rho_{Q}<3$. Precisely, we obtain an error term that involves the factor $K^{\frac{\rho_{Q}}{2}}$. The main difference is that the alternative proof works with the entries of the covariance matrices explicitly. A statement along the lines of Corollary 2 can be obtained analogously.

Theorem 4 (Convergence result for Algorithm 2) Assume that the trace class operator $Q$ is nonnegative and symmetric and $\left(W_{t}\right)_{t \geq 0}$ is a $Q$-Wiener process. Let $\Phi \in L(U, H)_{U_{0}}$ with $\left\|\Phi Q^{-\alpha}\right\|_{L_{H S}\left(U_{0}, H\right)}<C$ and $\Psi \in L\left(H, L\left(Q^{-\alpha} U, H\right)_{U_{0}}\right)$ for some $\alpha \in(0, \infty)$, i.e., assumptions (A1) and (A2) are fulfilled. Then, it holds

$$
\begin{aligned}
& \left(\mathrm{E}\left[\left\|\int_{t}^{t+h} \Psi\left(\int_{t}^{s} \Phi \mathrm{d} W_{r}\right) \mathrm{d} W_{s}-\sum_{i, j \in \mathcal{J}_{K}} \hat{I}_{(i, j)}^{Q,(D)}(h) \Psi\left(\Phi \tilde{e}_{i}, \tilde{e}_{j}\right)\right\|_{H}^{2}\right]\right)^{\frac{1}{2}} \\
& \quad \leq C\left(h\left(\sup _{j \in \mathcal{J} \backslash \mathcal{J}_{K}} \eta_{j}\right)^{\alpha}+\left(\max _{j \in \mathcal{J}_{K}} \eta_{j}\right)^{\frac{1}{2}}(\operatorname{tr} Q)^{\frac{3}{2}}\left(\min _{j \in \mathcal{J}_{K}} \eta_{j}\right)^{-\frac{1}{2}} \frac{h}{D}\right)
\end{aligned}
$$

for some $C>0$ and all $h>0, t, t+h \in[0, T], D, K \in \mathbb{N}$, and $\mathcal{J}_{K} \subset \mathcal{J}$ with $\left|\mathcal{J}_{K}\right|=K$.

Proof For a proof, we again refer to Sect. 3.

Remark 1 Note that if $\left(W_{t}\right)_{t \geq 0}$ is a cylindrical Wiener process, we get the same estimate (4) as in the finite dimensional case. 


\subsection{Comparison and concluding remarks}

For some fixed interval of length $h=\frac{T}{M}$ and a given projected $Q$-Wiener process $\left(W_{t}^{K}\right)_{t \geq 0}$, that is, some fixed values $M, K \in \mathbb{N}$, we obtain convergence as $D \rightarrow \infty$ for both algorithms that we proposed in the last sections with differing orders, respectively. However, if the approximation of the iterated integrals is incorporated in a numerical scheme to compute the solution of a given SPDE, then the interval length $h$ has to decrease and the number $K$, which defines the approximation of the $Q$-Wiener process, has to increase in order to approximate the solution with increasing accuracy. Algorithm 1 provides convergence as $K, M \rightarrow \infty$ if we choose $D \geq h^{2-2 \theta}$ for some $\theta>0$ with order $\mathcal{O}\left(M^{-\theta}\right)$ where the choice of $D$ does not depend on $K$. For Algorithm 2 , we require $D \geq\left(\min _{j \in \mathcal{J}_{K}} \eta_{j}\right)^{-\frac{1}{2}} h^{1-\theta}$ or, respectively, $D \geq \sqrt{K^{2}(K-1)} h^{1-\theta}$ for some $\theta>0$ to get convergence with order $\mathcal{O}\left(M^{-\theta}\right)$. In order to maintain the global rate of convergence in the mean-square sense in $h=\frac{T}{M}$ for a given numerical scheme, we need an even more careful choice of $D$. The error estimates for the iterated integrals depend on the values $M, D$, and, for Algorithm 2, on the value $K$. Moreover, for the approximation of the solution of an SPDE, we have to include an approximation of the infinite dimensional solution space, see [2], for example. All these error terms, i.e., in time, in space, the truncation of the $Q$-Wiener process and the truncation of the Fouries series for the iterated integrals, have to be balanced such that an optimal overall order of convergence for a given numerical scheme can be observed. The full treatment of this optimization is beyond the scope of this work as it, e.g., depends on the numerical scheme and the specific SPDE. We restrict our discussion to the clarification of the relation of the value $D$ to the number of time steps and the approximation of the $Q$ Wiener process which characterizes the main difference between the two algorithms that we proposed. However, this already shows that, dependent on the desired order of convergence in $h$ and on the operator $Q$ (which are determined by the SPDE to be solved and the numerical scheme employed for the discretization of time), either Algorithm 1 or Algorithm 2 is the optimal choice in terms of lower computational cost.

\section{Proofs}

\subsection{Convergence analysis for Algorithm 1}

Proof of Theorem 1 We determine the error resulting from the approximation of the iterated stochastic integral (7) by Algorithm 1 which also contains the projection of the $Q$-Wiener process in (9). Below, we employ error estimates of the following form several times, see also the proof in [2]. It holds 


$$
\begin{aligned}
\mathrm{E}\left[\left\|\int_{t}^{t+h} \Phi \mathrm{d}\left(W_{s}-W_{s}^{K}\right)\right\|_{H}^{2}\right] & =\mathrm{E}\left[\left\|\sum_{j \in \mathcal{J} \backslash \mathcal{J}_{K}} \int_{t}^{t+h} \Phi \tilde{e}_{j}\left\langle\mathrm{~d} W_{s}, \tilde{e}_{j}\right\rangle_{U}\right\|_{H}^{2}\right] \\
& =\sum_{j \in \mathcal{J} \backslash \mathcal{J}_{K}} \eta_{j} \int_{t}^{t+h} \mathrm{E}\left[\left\|\Phi Q^{-\alpha} Q^{\alpha} \tilde{e}_{j}\right\|_{H}^{2}\right] \mathrm{d} s \\
& =\sum_{j \in \mathcal{J} \backslash \mathcal{J}_{K}} \eta_{j}^{2 \alpha+1} \int_{t}^{t+h} \mathrm{E}\left[\left\|\Phi Q^{-\alpha} \tilde{e}_{j}\right\|_{H}^{2}\right] \mathrm{d} s \\
& \leq\left(\sup _{j \in \mathcal{J} \backslash \mathcal{J}_{K}} \eta_{j}\right)^{2 \alpha} \int_{t}^{t+h} \mathrm{E}\left[\sum \eta_{j \in \mathcal{J}}\left\|\Phi Q^{-\alpha} \tilde{e}_{j}\right\|_{H}^{2}\right] \mathrm{d} s \\
& =\left(\sup _{j \in \mathcal{J} \backslash \mathcal{J}_{K}} \eta_{j}\right)^{2 \alpha} \int_{t}^{t+h} \mathrm{E}\left[\left\|\Phi Q^{-\alpha}\right\|_{L_{H S}\left(U_{0}, H\right)}^{2}\right] \mathrm{d} s,
\end{aligned}
$$

where we used the expression

$$
\mathrm{d}\left(W_{s}-W_{s}^{K}\right)=\sum_{j \in \mathcal{J} \backslash \mathcal{J}_{K}} \tilde{e}_{j}\left\langle\mathrm{~d} W_{s}, \tilde{e}_{j}\right\rangle_{U}
$$

for all $s \in[0, T], K \in \mathbb{N}$ in the first step. We fix some arbitrary $h>0, t, t+h \in[0, T]$, and $K \in \mathbb{N}$ throughout the proof and decompose the error into several parts

$$
\begin{aligned}
& \mathrm{E}\left[\left\|\int_{t}^{t+h} \Psi\left(\int_{t}^{s} \Phi \mathrm{d} W_{r}\right) \mathrm{d} W_{s}-\sum_{i, j \in \mathcal{J}_{K}} \bar{I}_{(i, j)}^{Q,(D)}(h) \Psi\left(\Phi \tilde{e}_{i}, \tilde{e}_{j}\right)\right\|_{H}^{2}\right] \\
& \leq C\left(\mathrm{E}\left[\left\|\int_{t}^{t+h} \Psi\left(\int_{t}^{s} \Phi \mathrm{d} W_{r}\right) \mathrm{d} W_{s}-\int_{t}^{t+h} \Psi\left(\int_{t}^{s} \Phi \mathrm{d} W_{r}^{K}\right) \mathrm{d} W_{s}\right\|_{H}^{2}\right]\right. \\
&+\mathrm{E}\left[\left\|\int_{t}^{t+h} \Psi\left(\int_{t}^{s} \Phi \mathrm{d} W_{r}^{K}\right) \mathrm{d} W_{s}-\int_{t}^{t+h} \Psi\left(\int_{t}^{s} \Phi \mathrm{d} W_{r}^{K}\right) \mathrm{d} W_{s}^{K}\right\|_{H}^{2}\right] \\
&\left.+\mathrm{E}\left[\left\|\int_{t}^{t+h} \Psi\left(\int_{t}^{s} \Phi \mathrm{d} W_{r}^{K}\right) \mathrm{d} W_{s}^{K}-\sum_{i, j \in \mathcal{J}_{K}} \bar{I}_{(i, j)}^{Q,(D)}(h) \Psi\left(\Phi \tilde{e}_{i}, \tilde{e}_{j}\right)\right\|_{H}^{2}\right]\right) .
\end{aligned}
$$

For now, we neglect the last term in (29) and estimate the other parts. By Itô's isometry, the properties (A1) and (A2) of the operators $\Phi, \Psi$, and estimate (28), we get

$$
\begin{aligned}
& \mathrm{E}\left[\left\|\int_{t}^{t+h} \Psi\left(\int_{t}^{s} \Phi \mathrm{d} W_{r}\right) \mathrm{d} W_{s}-\int_{t}^{t+h} \Psi\left(\int_{t}^{s} \Phi \mathrm{d} W_{r}^{K}\right) \mathrm{d} W_{s}\right\|_{H}^{2}\right] \\
&+\mathrm{E}\left[\left\|\int_{t}^{t+h} \Psi\left(\int_{t}^{s} \Phi \mathrm{d} W_{r}^{K}\right) \mathrm{d} W_{s}-\int_{t}^{t+h} \Psi\left(\int_{t}^{s} \Phi \mathrm{d} W_{r}^{K}\right) \mathrm{d} W_{s}^{K}\right\|_{H}^{2}\right] \\
& \leq \int_{t}^{t+h} \mathrm{E}\left[\left\|\Psi\left(\int_{t}^{s} \Phi \mathrm{d}\left(W_{r}-W_{r}^{K}\right)\right)\right\|_{L_{H S}\left(U_{0}, H\right)}^{2}\right] \mathrm{d} s
\end{aligned}
$$




$$
\begin{aligned}
& +\left(\sup _{j \in \mathcal{J} \backslash \mathcal{J}_{K}} \eta_{j}\right)^{2 \alpha} \int_{t}^{t+h} \mathrm{E}\left[\left\|\Psi\left(\int_{t}^{s} \Phi \mathrm{d} W_{r}^{K}\right) Q^{-\alpha}\right\|_{L_{H S}\left(U_{0}, H\right)}^{2}\right] \mathrm{d} s \\
& \leq C \int_{t}^{t+h} \mathrm{E}\left[\left\|\int_{t}^{s} \Phi \mathrm{d}\left(W_{r}-W_{r}^{K}\right)\right\|_{H}^{2}\right] \mathrm{d} s \\
& +C\left(\sup _{j \in \mathcal{J} \backslash \mathcal{J}_{K}} \eta_{j}\right)^{2 \alpha} \int_{t}^{t+h} \mathrm{E}\left[\left\|\int_{t}^{s} \Phi \mathrm{d} W_{r}^{K}\right\|_{H}^{2}\right] \mathrm{d} s \\
& \leq C\left(\sup _{j \in \mathcal{J} \backslash \mathcal{J}_{K}} \eta_{j}\right)^{2 \alpha} \int_{t}^{t+h} \int_{t}^{s} \mathrm{E}\left[\left\|\Phi Q^{-\alpha}\right\|_{L_{H S}\left(U_{0}, H\right)}^{2}\right] \mathrm{d} r \mathrm{~d} s \\
& +C\left(\sup _{j \in \mathcal{J} \backslash \mathcal{J}_{K}} \eta_{j}\right)^{2 \alpha} \int_{t}^{t+h} \int_{t}^{s} \mathrm{~d} r \mathrm{~d} s .
\end{aligned}
$$

Finally, assumption (A1) yields

$$
\begin{aligned}
\mathrm{E}\left[\left\|\int_{t}^{t+h} \Psi\left(\int_{t}^{s} \Phi \mathrm{d} W_{r}\right) \mathrm{d} W_{s}-\int_{t}^{t+h} \Psi\left(\int_{t}^{s} \Phi \mathrm{d} W_{r}^{K}\right) \mathrm{d} W_{s}\right\|_{H}^{2}\right] \\
\quad+\mathrm{E}\left[\left\|\int_{t}^{t+h} \Psi\left(\int_{t}^{s} \Phi \mathrm{d} W_{r}^{K}\right) \mathrm{d} W_{s}-\int_{t}^{t+h} \Psi\left(\int_{t}^{s} \Phi \mathrm{d} W_{r}^{K}\right) \mathrm{d} W_{s}^{K}\right\|_{H}^{2}\right] \\
\quad \leq C\left(\sup _{j \in \mathcal{J} \backslash \mathcal{J}_{K}} \eta_{j}\right)^{2 \alpha} h^{2} .
\end{aligned}
$$

Now, we concentrate on the last term in (29); this part also proves Corollary 1 . We get

$$
\begin{aligned}
& \mathrm{E}\left[\left\|\int_{t}^{t+h} \Psi\left(\int_{t}^{s} \Phi \mathrm{d} W_{r}^{K}\right) \mathrm{d} W_{s}^{K}-\sum_{i, j \in \mathcal{J}_{K}} \bar{I}_{(i, j)}^{Q,(D)}(h) \Psi\left(\Phi \tilde{e}_{i}, \tilde{e}_{j}\right)\right\|_{H}^{2}\right] \\
& =\mathrm{E}\left[\left\|\sum_{i, j \in \mathcal{J}_{K}} I_{(i, j)}^{Q}(h) \Psi\left(\Phi \tilde{e}_{i}, \tilde{e}_{j}\right)-\sum_{i, j \in \mathcal{J}_{K}} \bar{I}_{(i, j)}^{Q,(D)}(h) \Psi\left(\Phi \tilde{e}_{i}, \tilde{e}_{j}\right)\right\|_{H}^{2}\right] \\
& =\sum_{i, j \in \mathcal{J}_{K}} \mathrm{E}\left[\left(I_{(i, j)}^{Q}(h)-\bar{I}_{(i, j)}^{Q,(D)}(h)\right)^{2}\right]\left\|\Psi\left(\Phi \tilde{e}_{i}, \tilde{e}_{j}\right)\right\|_{H}^{2}
\end{aligned}
$$

as $\mathrm{E}\left[\left(I_{(i, j)}^{Q}(h)-\bar{I}_{(i, j)}^{Q,(D)}(h)\right)\left(I_{(k, l)}^{Q}(h)-\bar{I}_{(k, l)}^{Q,(D)}(h)\right)\right]=0$ for all $i, j, k, l \in \mathcal{J}_{K}$ with $(i, j) \neq(k, l), K \in \mathbb{N}$, see [3]. By assumptions (B1) and (B2), we obtain

$$
\begin{aligned}
\mathrm{E}\left[\left\|\int_{t}^{t+h} \Psi\left(\int_{t}^{s} \Phi \mathrm{d} W_{r}^{K}\right) \mathrm{d} W_{s}^{K}-\sum_{i, j \in \mathcal{J}_{K}} \bar{I}_{(i, j)}^{Q,(D)}(h) \Psi\left(\Phi \tilde{e}_{i}, \tilde{e}_{j}\right)\right\|_{H}^{2}\right] \\
\leq \sum_{i, j \in \mathcal{J}_{K}} \mathrm{E}\left[\left(I_{(i, j)}^{Q}(h)-\bar{I}_{(i, j)}^{Q,(D)}(h)\right)^{2}\right]\|\Psi\|_{L(H, L(U, H))}^{2}\|\Phi\|_{L(U, H)}^{2} \\
\leq C \sum_{i, j \in \mathcal{J}_{K}} \mathrm{E}\left[\left(I_{(i, j)}^{Q}(h)-\bar{I}_{(i, j)}^{Q,(D)}(h)\right)^{2}\right] .
\end{aligned}
$$


Due to the relations (14)-(16), it is enough to examine $\tilde{A}^{Q}(h)$ and $\tilde{A}^{Q,(D)}(h)$ which implies

$$
\begin{aligned}
& \mathrm{E}\left[\left\|\int_{t}^{t+h} \Psi\left(\int_{t}^{s} \Phi \mathrm{d} W_{r}^{K}\right) \mathrm{d} W_{s}^{K}-\sum_{i, j \in \mathcal{J}_{K}} \bar{I}_{(i, j)}^{Q,(D)}(h) \Psi\left(\Phi \tilde{e}_{i}, \tilde{e}_{j}\right)\right\|_{H}^{2}\right] \\
& \quad \leq 2 C \sum_{i=1}^{L} \mathrm{E}\left[\left(\tilde{A}_{(i)}^{Q}(h)-\tilde{A}_{(i)}^{Q,(D)}(h)\right)^{2}\right] .
\end{aligned}
$$

By (12), (13), and the properties of $a_{r}^{j}, b_{r}^{j}$ for $r \in \mathbb{N}_{0}, j \in \mathcal{J}_{K}, K \in \mathbb{N}$, we obtain

$$
\begin{aligned}
\mathrm{E}\left[\left\|\int_{t}^{t+h} \Psi\left(\int_{t}^{s} \Phi \mathrm{d} W_{r}^{K}\right) \mathrm{d} W_{s}^{K}-\sum_{i, j \in \mathcal{J}_{K}} \bar{I}_{(i, j)}^{Q,(D)}(h) \Psi\left(\Phi \tilde{e}_{i}, \tilde{e}_{j}\right)\right\|_{H}^{2}\right] \\
\quad \leq 2 C \sum_{\substack{i, j \in \mathcal{J}_{K} \\
i<j}} \mathrm{E}\left[\left(\pi \sum_{r=D+1}^{\infty} r\left(a_{r}^{i}\left(b_{r}^{j}-\frac{1}{\pi r} \Delta w_{h}^{j}\right)-\left(b_{r}^{i}-\frac{1}{\pi r} \Delta w_{h}^{i}\right) a_{r}^{j}\right)\right)^{2}\right] \\
=2 C \pi^{2} \sum_{i, j \in \mathcal{J}_{K}} \sum_{r=D+1}^{\infty} r^{2} \mathrm{E}\left[\left(a_{r}^{i} b_{r}^{j}-a_{r}^{i} \frac{1}{\pi r} \Delta w_{h}^{j}\right)^{2}+\left(b_{r}^{i} a_{r}^{j}-\frac{1}{\pi r} \Delta w_{h}^{i} a_{r}^{j}\right)^{2}\right] \\
=3 C \frac{h^{2}}{\pi^{2}} \sum_{\substack{i, j \in \mathcal{J}_{K} \\
i<j}} \eta_{i} \eta_{j} \sum_{r=D+1}^{\infty} \frac{1}{r^{2}} \\
\leq 3 C \frac{h^{2}}{\pi^{2}}(\operatorname{tr} Q)^{2} \sum_{r=D+1}^{\infty} \frac{1}{r^{2}}
\end{aligned}
$$

for all $D \in \mathbb{N}$. As in [4], we finally estimate

$$
\sum_{r=D+1}^{\infty} \frac{1}{r^{2}} \leq \int_{D}^{\infty} \frac{1}{s^{2}} \mathrm{~d} s=\frac{1}{D}
$$

and, in total, we obtain for this part

$$
\begin{aligned}
& \mathrm{E}\left[\left\|\int_{t}^{t+h} \Psi\left(\int_{t}^{s} \Phi \mathrm{d} W_{r}^{K}\right) \mathrm{d} W_{s}^{K}-\sum_{i, j \in \mathcal{J}_{K}} \bar{I}_{(i, j)}^{Q,(D)}(h) \Psi\left(\Phi \tilde{e}_{i}, \tilde{e}_{j}\right)\right\|_{H}^{2}\right] \\
& \quad \leq 3 C(\operatorname{tr} Q)^{2} \frac{h^{2}}{D \pi^{2}}
\end{aligned}
$$

for all $h>0, t, t+h \in[0, T], D, K \in \mathbb{N}$. 


\subsection{Square root of the covariance matrix}

Proof of Theorem 2 It holds $\Sigma_{\infty}^{Q}=\tilde{Q}_{K} \Sigma_{\infty}^{I} \tilde{Q}_{K}^{T}$, where $\Sigma_{\infty}^{I}$ is given by (23) for $Q_{K}=$ $I_{K}$, and $\Delta w_{h}^{I}=Q_{K}^{-1 / 2} \Delta w_{h}^{Q}=\sqrt{h} V$. We assume that

$$
\sqrt{\Sigma_{\infty}^{Q}}=\tilde{Q}_{K} \frac{\Sigma_{\infty}^{I}+2 \sqrt{1+V^{T} V} I_{L}}{\sqrt{2}\left(1+\sqrt{1+V^{T} V}\right)}=\tilde{Q}_{K} \frac{\Sigma_{\infty}^{I}+2 \sqrt{1+\frac{1}{h} \Delta w_{h}^{I} \Delta w_{h}^{I}} I_{L}}{\sqrt{2}\left(1+\sqrt{1+\frac{1}{h} \Delta w_{h}^{I^{T}} \Delta w_{h}^{I}}\right)}
$$

holds and compute for $a:=\sqrt{1+\frac{1}{h} \Delta w_{h}^{I^{T}} \Delta w_{h}^{I}}$ the expression

$$
\begin{aligned}
\sqrt{\Sigma_{\infty}^{Q}} \sqrt{\Sigma_{\infty}^{Q}} & \\
= & \frac{\tilde{Q}_{K} \Sigma_{\infty}^{I}\left(\Sigma_{\infty}^{I}\right)^{T} \tilde{Q}_{K}^{T}+2 a \tilde{Q}_{K} \Sigma_{\infty}^{I} \tilde{Q}_{K}^{T}+2 a \tilde{Q}_{K}\left(\Sigma_{\infty}^{I}\right)^{T} \tilde{Q}_{K}^{T}+4 a^{2} \tilde{Q}_{K} \tilde{Q}_{K}^{T}}{2(1+a)^{2}} \\
= & \frac{\tilde{Q}_{K} \Sigma_{\infty}^{I}\left(\Sigma_{\infty}^{I}\right)^{T} \tilde{Q}_{K}^{T}-\left(2+2 a^{2}\right) \tilde{Q}_{K} \Sigma_{\infty}^{I} \tilde{Q}_{K}^{T}+4 a^{2} \tilde{Q}_{K} \tilde{Q}_{K}^{T}}{2(1+a)^{2}} \\
& +\frac{2+4 a+2 a^{2}}{2(1+a)^{2}} \tilde{Q}_{K} \Sigma_{\infty}^{I} \tilde{Q}_{K}^{T} \\
= & \frac{\tilde{Q}_{K} \Sigma_{\infty}^{I}\left(\Sigma_{\infty}^{I}\right)^{T} \tilde{Q}_{K}^{T}-\left(2+2 a^{2}\right) \tilde{Q}_{K} \Sigma_{\infty}^{I} \tilde{Q}_{K}^{T}+4 a^{2} \tilde{Q}_{K} \tilde{Q}_{K}^{T}+\Sigma_{\infty}^{Q} .}{2(1+a)^{2}}
\end{aligned}
$$

The idea in [9] is to show that the first term, which slightly differs in [9], is zero, i.e.,

$$
\begin{array}{r}
\tilde{Q}_{K}\left(\Sigma_{\infty}^{I}\left(\Sigma_{\infty}^{I}\right)^{T}-\left(2+2 a^{2}\right) \Sigma_{\infty}^{I}+4 a^{2} I_{L}\right) \tilde{Q}_{K}^{T}=0_{L \times L} \\
\Leftrightarrow \Sigma_{\infty}^{I}\left(\Sigma_{\infty}^{I}\right)^{T}-\left(2+2 a^{2}\right) \Sigma_{\infty}^{I}+4 a^{2} I_{L}=0_{L \times L},
\end{array}
$$

which proves that the expression for $\sqrt{\Sigma_{\infty}^{Q}}$ is correct. In the proof of Theorem 4.1 in [9], the author shows

$$
\Sigma_{\infty}^{I}\left(\Sigma_{\infty}^{I}\right)^{T}-\left(2+2 a^{2}\right) \Sigma_{\infty}^{I}+4 a^{2} I_{L}=0_{L \times L}
$$

arguing by the eigenvalues of the minimal polynomial of this equation. We do not repeat this ideas here but refer to [9] for further details.

\subsection{Convergence analysis for Algorithm 2}

Proof of Theorem 3 We split the error term as in the proof of Theorem 1, see Eq. (29), and obtain the same expression (30) from the approximation of the $Q$-Wiener process by $\left(W_{t}^{K}\right)_{t \geq 0}, K \in \mathbb{N}$. Further, we get as in Eq. (31) 


$$
\begin{aligned}
& \mathrm{E}\left[\left\|\int_{t}^{t+h} \Psi\left(\int_{t}^{s} \Phi \mathrm{d} W_{r}^{K}\right) \mathrm{d} W_{s}^{K}-\sum_{i, j \in \mathcal{J}_{K}} \hat{I}_{(i, j)}^{Q,(D)}(h) \Psi\left(\Phi \tilde{e}_{i}, \tilde{e}_{j}\right)\right\|_{H}^{2}\right] \\
& \quad \leq 2 C \sum_{i=1}^{L} \mathrm{E}\left[\left(\tilde{A}_{(i)}^{Q}(h)-\hat{A}_{(i)}^{Q,(D)}(h)\right)^{2}\right]
\end{aligned}
$$

for all $h>0, t, t+h \in[0, T], K \in \mathbb{N}$. The following part also proves Corollary 2 . Let $\|\cdot\|_{F}$ denote the Frobenius norm. With the expressions for $\tilde{R}^{Q,(D)}(h)$ in $(25)$, with $\Sigma^{Q,(D)}=\tilde{Q}_{K} \Sigma^{I,(D)} \tilde{Q}_{K}^{T}, \Sigma_{\infty}^{Q}=\tilde{Q}_{K} \Sigma_{\infty}^{I} \tilde{Q}_{K}^{T}$ where $\Sigma^{I,(D)}, \Sigma_{\infty}^{I}$ are given by (26) and (23) for $Q_{K}=I_{K}$, respectively, and the definition of the algorithm (27), we obtain

$$
\begin{aligned}
& \mathrm{E}\left[\left\|\int_{t}^{t+h} \Psi\left(\int_{t}^{s} \Phi \mathrm{d} W_{r}^{K}\right) \mathrm{d} W_{s}^{K}-\sum_{i, j \in \mathcal{J}_{K}} \hat{I}_{(i, j)}^{Q,(D)}(h) \Psi\left(\Phi \tilde{e}_{i}, \tilde{e}_{j}\right)\right\|_{H}^{2}\right] \\
& \leq 2 C \sum_{i=1}^{L} \mathrm{E}\left[\left(\left(\tilde{R}^{Q,(D)}(h)-\frac{h}{2 \pi}\left(\sum_{r=D+1}^{\infty} \frac{1}{r^{2}}\right)^{\frac{1}{2}} \sqrt{\Sigma_{\infty}^{Q}} \Upsilon^{Q,(D)}\right)_{(i)}\right)^{2}\right] \\
& =2 C \sum_{i=1}^{L} \mathrm{E}\left[\left(\left(\frac{h}{2 \pi}\left(\sum_{r=D+1}^{\infty} \frac{1}{r^{2}} H_{K} \Sigma^{Q}\left(V_{r}^{Q}\right)_{\mid Z_{r}^{Q}, \Delta w_{h}^{Q}} H_{K}^{T}\right)^{\frac{1}{2}} \Upsilon^{Q,(D)}\right.\right.\right.
\end{aligned}
$$

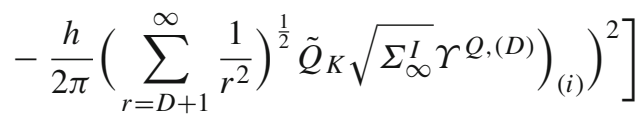

$$
\begin{aligned}
& =\frac{C h^{2}}{2 \pi^{2}}\left(\sum_{r=D+1}^{\infty} \frac{1}{r^{2}}\right) \sum_{i=1}^{L} \mathrm{E}\left[\left(\left(\left(\sum_{r=D+1}^{\infty} \frac{1}{r^{2}}\right)^{-\frac{1}{2}}\right.\right.\right. \\
& \left.\left.\left.\left.\times \tilde{Q}_{K}\left(\sum_{r=D+1}^{\infty} \frac{1}{r^{2}} H_{K} \Sigma^{I}\left(V_{r}^{I}\right)_{\mid Z_{r}^{I}, \Delta w_{h}^{I}} H_{K}^{T}\right)^{\frac{1}{2}}-\tilde{Q}_{K} \sqrt{\Sigma_{\infty}^{I}}\right) \Upsilon^{Q,(D)}\right)_{(i)}\right)^{2}\right] \\
& =C \frac{h^{2}}{2 \pi^{2}}\left(\sum_{r=D+1}^{\infty} \frac{1}{r^{2}}\right) \sum_{i=1}^{L} \mathrm{E}\left[\left(\left(\left(\tilde{Q}_{K}\left(\sqrt{\Sigma^{I,(D)}}-\sqrt{\Sigma_{\infty}^{I}}\right)\right) \Upsilon^{Q,(D)}\right)_{(i)}\right)^{2}\right] \\
& =C \frac{h^{2}}{2 \pi^{2}}\left(\sum_{r=D+1}^{\infty} \frac{1}{r^{2}}\right) \\
& \times \sum_{i=1}^{L} \mathrm{E}\left[\mathrm{E}\left[\left(\left(\left(\tilde{Q}_{K}\left(\sqrt{\Sigma^{I,(D)}}-\sqrt{\Sigma_{\infty}^{I}}\right)\right) \Upsilon^{Q,(D)}\right)_{(i)}\right)^{2} \mid Z^{Q}, \Delta w_{h}^{Q}\right]\right] \\
& =C \frac{h^{2}}{2 \pi^{2}}\left(\sum_{r=D+1}^{\infty} \frac{1}{r^{2}}\right) \mathrm{E}\left[\left\|\tilde{Q}_{K}\left(\sqrt{\Sigma^{I,(D)}}-\sqrt{\Sigma_{\infty}^{I}}\right)\right\|_{F}^{2}\right]
\end{aligned}
$$

for all $h>0, t, t+h \in[0, T], D, K \in \mathbb{N}$. Here, we used that $\Upsilon^{Q,(D)}{ }_{\mid Z Q}{ }_{\mid, \Delta w_{h}^{Q}} \sim$ $N\left(0_{L}, I_{L}\right)$ for $h>0, D, L \in \mathbb{N}$ and that $\tilde{Q}_{K}$ is a diagonal matrix. Precisely, for 
$G:=\sqrt{\Sigma^{I,(D)}}-\sqrt{\Sigma_{\infty}^{I}}$ with $G:=\left(g_{i j}\right)_{1 \leq i, j \leq L}$ and $\Upsilon^{Q,(D)}=\left(\Upsilon_{j}^{Q,(D)}\right)_{1 \leq j \leq L}$, we compute

$$
\begin{aligned}
& \left.\sum_{i=1}^{L} \mathrm{E}\left[\mathrm{E}\left[\left(\left(\tilde{Q}_{K}\left(\sqrt{\Sigma^{I,(D)}}-\sqrt{\Sigma_{\infty}^{I}}\right)\right) \Upsilon^{Q,(D)}\right)_{(i)}\right)^{2} \mid Z^{Q}, \Delta w_{h}^{Q}\right]\right] \\
& =\sum_{i=1}^{L} \mathrm{E}\left[\mathrm{E}\left[\left(\left(\tilde{Q}_{K} G \Upsilon^{Q,(D)}\right)_{(i)}\right)^{2} \mid Z^{Q}, \Delta w_{h}^{Q}\right]\right] \\
& =\sum_{i=1}^{L} \mathrm{E}\left[\mathrm{E}\left[\left(\sum_{j=1}^{L}\left(\tilde{Q}_{K}\right)_{i i} g_{i j} \Upsilon_{j}^{Q,(D)}\right)^{2} \mid Z^{Q}, \Delta w_{h}^{Q}\right]\right] \\
& =\sum_{i, j=1}^{L} \mathrm{E}\left[\left(\tilde{Q}_{K}\right)_{i i}^{2} g_{i j}^{2}\right] \\
& =\mathrm{E}\left[\left\|\tilde{Q}_{K} G\right\|_{F}^{2}\right] \\
& =\mathrm{E}\left[\left\|\tilde{Q}_{K}\left(\sqrt{\Sigma^{I,(D)}}-\sqrt{\Sigma_{\infty}^{I}}\right)\right\|_{F}^{2}\right] .
\end{aligned}
$$

In order to relate to the proof in [9], we write

$$
\begin{aligned}
\mathrm{E}\left[\left\|\tilde{Q}_{K}\left(\sqrt{\Sigma^{I,(D)}}-\sqrt{\Sigma_{\infty}^{I}}\right)\right\|_{F}^{2}\right] & =\mathrm{E}\left[\sum_{i, j=1}^{L}\left(\tilde{Q}_{K}\right)_{i i}^{2} g_{i j}^{2}\right] \\
& \leq \max _{j \in \mathcal{J}_{K}} \eta_{j}^{2} \mathrm{E}\left[\sum_{i, j=1}^{L} g_{i j}^{2}\right] \\
& \leq \max _{j \in \mathcal{J}_{K}} \eta_{j}^{2} \mathrm{E}\left[\left\|\sqrt{\Sigma^{I,(D)}}-\sqrt{\Sigma_{\infty}^{I}}\right\|_{F}^{2}\right]
\end{aligned}
$$

In total, we obtain

$$
\begin{aligned}
& \mathrm{E}\left[\left\|\int_{t}^{t+h} \Psi\left(\int_{t}^{s} \Phi \mathrm{d} W_{r}^{K}\right) \mathrm{d} W_{s}^{K}-\sum_{i, j \in \mathcal{J}_{K}} \hat{I}_{(i, j)}^{Q,(D)}(h) \Psi\left(\Phi \tilde{e}_{i}, \tilde{e}_{j}\right)\right\|_{H}^{2}\right] \\
& \leq C \max _{j \in \mathcal{J}_{K}} \eta_{j}^{2} \frac{h^{2}}{2 \pi^{2}}\left(\sum_{r=D+1}^{\infty} \frac{1}{r^{2}}\right) \mathrm{E}\left[\left\|\sqrt{\Sigma^{I,(D)}}-\sqrt{\Sigma_{\infty}^{I}}\right\|_{F}^{2}\right] .
\end{aligned}
$$

Now, we can insert the results obtained in the proofs of [9, Theorem 4.1, Theorem 4.2, Theorem 4.3]; this yields 


$$
\begin{aligned}
& \mathrm{E}\left[\left\|\int_{t}^{t+h} \Psi\left(\int_{t}^{s} \Phi \mathrm{d} W_{r}^{K}\right) \mathrm{d} W_{s}^{K}-\sum_{i, j \in \mathcal{J}_{K}} \hat{I}_{(i, j)}^{Q,(D)}(h) \Psi\left(\Phi \tilde{e}_{i}, \tilde{e}_{j}\right)\right\|_{H}^{2}\right] \\
& \leq C \max _{j \in \mathcal{J}_{K}} \eta_{j}^{2} \frac{h^{2} K(K-1)\left(K+4 \mathrm{E}\left[V^{T} V\right]\right)}{12 \pi^{2} D^{2}} \\
& \leq C \max _{j \in \mathcal{J}_{K}} \eta_{j}^{2} \frac{5 h^{2} K^{2}(K-1)}{12 \pi^{2} D^{2}}
\end{aligned}
$$

for all $h>0, t, t+h \in[0, T], D, K \in \mathbb{N}$ where $V=h^{-1 / 2} Q_{K}^{-1 / 2} \Delta w_{h}^{Q}$.

Proof of Theorem 4 We split the error term as in the proof of Theorems 1 and 3, see Eq. (29), and obtain the same expression (30) from the approximation of the $Q$-Wiener process by $\left(W_{t}^{K}\right)_{t \geq 0}, K \in \mathbb{N}$. Moreover, as in the previous proof, we get from (33) that

$$
\begin{aligned}
& \mathrm{E}\left[\left\|\int_{t}^{t+h} \Psi\left(\int_{t}^{s} \Phi \mathrm{d} W_{r}^{K}\right) \mathrm{d} W_{s}^{K}-\sum_{i, j \in \mathcal{J}_{K}} \hat{I}_{(i, j)}^{Q,(D)}(h) \Psi\left(\Phi \tilde{e}_{i}, \tilde{e}_{j}\right)\right\|_{H}^{2}\right] \\
& \leq C \frac{h^{2}}{2 \pi^{2}}\left(\sum_{r=D+1}^{\infty} \frac{1}{r^{2}}\right) \mathrm{E}\left[\| \sqrt{\Sigma^{Q,(D)}}-\sqrt{\Sigma_{\infty}^{Q} \|_{F}^{2}}\right]
\end{aligned}
$$

for all $h>0, t, t+h \in[0, T], D, K \in \mathbb{N}$. In this alternative proof, we consider the elements of the matrices $\Sigma^{Q,(D)}$ and $\Sigma_{\infty}^{Q}$ explicitly. Therefore, we define the index set of interest as $\mathcal{I}_{A}=((1,2), \ldots,(1, K), \ldots,(l, l+1), \ldots,(l, K), \ldots,(K-1, K))=$ $\left(I_{1}, \ldots, I_{L}\right)$ which selects the same entries of some matrix as the matrix transformation by $H_{K}$ given in (17). The $L \times L$-matrix $H_{K} \Sigma^{Q}\left(V_{1}^{Q}\right)_{\mid Z_{1}^{Q}, \Delta w_{h}^{Q}} H_{K}^{T}$ has entries of type

$$
\begin{aligned}
& \mathrm{E}\left[\left(U_{1 i}^{Q}\left(Z_{1 j}^{Q}-\sqrt{\frac{2}{h} \Delta w_{h}^{j}}\right)-\left(Z_{1 i}^{Q}-\sqrt{\left.\left.\frac{2}{h} \Delta w_{h}^{i}\right) U_{1 j}^{Q}\right)}\right.\right.\right. \\
& \left.\quad \times\left(U_{1 m}^{Q}\left(Z_{1 n}^{Q}-\sqrt{\frac{2}{h}} \Delta w_{h}^{n}\right)-\left(Z_{1 m}^{Q}-\sqrt{\frac{2}{h}} \Delta w_{h}^{m}\right) U_{1 n}^{Q}\right) \mid Z_{1}^{Q}, \Delta w_{h}^{Q}\right]
\end{aligned}
$$

for some $i, j, m, n \in\{1, \ldots, K\}$ with $i<j$ and $m<n$. Especially, its diagonal entries are of type

$$
\eta_{i}\left(Z_{1 j}^{Q}-\sqrt{\frac{2}{h}} \Delta w_{h}^{j}\right)^{2}+\eta_{j}\left(Z_{1 i}^{Q}-\sqrt{\frac{2}{h}} \Delta w_{h}^{i}\right)^{2}
$$

with $(i, j) \in \mathcal{I}_{A}$ and $i \neq j$. The off-diagonal entries of $H_{K} \Sigma^{Q}\left(V_{1}^{Q}\right)_{\mid Z_{1}^{Q}, \Delta w_{h}^{Q}} H_{K}^{T}$ are of the form 


$$
\begin{aligned}
& \mathrm{E}\left[\left(U_{1 i}^{Q}\left(Z_{1 j}^{Q}-\sqrt{\frac{2}{h}} \Delta w_{h}^{j}\right)-\left(Z_{1 i}^{Q}-\sqrt{\frac{2}{h}} \Delta w_{h}^{i}\right) U_{1 j}^{Q}\right)\right. \\
& \left.\times\left(U_{1 m}^{Q}\left(Z_{1 n}^{Q}-\sqrt{\frac{2}{h}} \Delta w_{h}^{n}\right)-\left(Z_{1 m}^{Q}-\sqrt{\frac{2}{h}} \Delta w_{h}^{m}\right) U_{1 n}^{Q}\right) \mid Z_{1}^{Q}, \Delta w_{h}^{Q}\right] \\
& =\left\{\begin{array}{cc}
0, & i, j \notin\{m, n\} \\
\eta_{i}\left(Z_{1 j}^{Q}-\sqrt{\frac{2}{h}} \Delta w_{h}^{j}\right)\left(Z_{1 n}^{Q}-\sqrt{\frac{2}{h}} \Delta w_{h}^{n}\right), & i=m, j \neq n \\
-\eta_{i}\left(Z_{1 j}^{Q}-\sqrt{\frac{2}{h}} \Delta w_{h}^{j}\right)\left(Z_{1 m}^{Q}-\sqrt{\frac{2}{h}} \Delta w_{h}^{m}\right), i=n, j \neq m \\
-\eta_{j}\left(Z_{1 i}^{Q}-\sqrt{\frac{2}{h}} \Delta w_{h}^{i}\right)\left(Z_{1 n}^{Q}-\sqrt{\frac{2}{h}} \Delta w_{h}^{n}\right), \quad j=m, i \neq n \\
\eta_{j}\left(Z_{1 i}^{Q}-\sqrt{\frac{2}{h}} \Delta w_{h}^{i}\right)\left(Z_{1 m}^{Q}-\sqrt{\frac{2}{h}} \Delta w_{h}^{m}\right), j=n, i \neq m
\end{array}\right.
\end{aligned}
$$

with $i, j, m, n \in\{1, \ldots, K\}, i<j$ and $m<n$. Therewith, it is easy to see that for

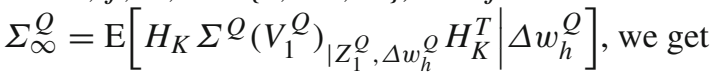

$$
\left(\Sigma_{\infty}^{Q}\right)_{(k, k)}=2 \eta_{i} \eta_{j}+\frac{2}{h} \eta_{i}\left(\Delta w_{h}^{j}\right)^{2}+\frac{2}{h} \eta_{j}\left(\Delta w_{h}^{i}\right)^{2}
$$

and for the off-diagonal entries, it holds

$$
\left(\Sigma_{\infty}^{Q}\right)_{(k, l)}= \begin{cases}0, & i, j \notin\{m, n\} \\ \frac{2}{h} \eta_{i} \Delta w_{h}^{j} \Delta w_{h}^{n}, & i=m, j \neq n \\ -\frac{2}{h} \eta_{i} \Delta w_{h}^{j} \Delta w_{h}^{m}, & i=n, j \neq m \\ -\frac{2}{h} \eta_{j} \Delta w_{h}^{i} \Delta w_{h}^{n}, & j=m, i \neq n \\ \frac{2}{h} \eta_{j} \Delta w_{h}^{i} \Delta w_{h}^{m}, & j=n, i \neq m\end{cases}
$$

with $k, l \in\{1, \ldots, L\}, l \neq k, i, j, m, n \in\{1, \ldots, K\}, i<j$ and $m<n$. Next, we employ the following lemma from [9] in order to rewrite (34).

Lemma 1 Let $A$ and $G$ be symmetric positive definite matrices and denote the smallest eigenvalue of matrix $G$ by $\lambda_{\text {min }}$. Then, it holds

$$
\left\|A^{\frac{1}{2}}-G^{\frac{1}{2}}\right\|_{F}^{2} \leq \frac{1}{\sqrt{\lambda_{\min }}}\|A-G\|_{F}^{2} .
$$

Proof of Lemma 1 A proof can be found in [9, Lemma 4.1].

For simplicity, we assume $\eta_{1} \geq \eta_{2} \geq \ldots \geq \eta_{K}$ for all $K \in \mathbb{N}$. We decompose $\Sigma_{\infty}^{Q}$ as

$$
\Sigma_{\infty}^{Q}=2 \eta_{K-1} \eta_{K} I_{L}+\widehat{\Sigma_{\infty}^{Q}}
$$

to determine its smallest eigenvalue. The matrix $\widehat{\Sigma_{\infty}^{Q}}$ is defined as follows: For the diagonal elements, we get values 


$$
\begin{aligned}
\widehat{\left(\Sigma_{\infty}^{Q}\right)_{(k, k)}} & =\left(\Sigma_{\infty}^{Q}\right)_{(k, k)}-2 \eta_{K-1} \eta_{K} \\
& =2\left(\eta_{i} \eta_{j}-\eta_{K-1} \eta_{K}\right)+\frac{2}{h} \eta_{i}\left(\Delta w_{h}^{j}\right)^{2}+\frac{2}{h} \eta_{j}\left(\Delta w_{h}^{i}\right)^{2} \geq 0
\end{aligned}
$$

with $k \in\{1, \ldots, L\},(i, j) \in \mathcal{I}_{A}$, and $h>0$. For the off-diagonal elements, we get $\widehat{\left(\Sigma_{\infty}^{Q}\right)_{(k, l)}}=\left(\Sigma_{\infty}^{Q}\right)_{(k, l)}$ for all $k, l \in\{1, \ldots, L\}, k \neq l$. As the matrix $\widehat{\Sigma_{\infty}^{Q}}$ is symmetric and positive semi-definite, the smallest eigenvalue $\lambda_{\min }$ of $\Sigma_{\infty}^{Q}$ fulfills $\lambda_{\min } \geq 2 \eta_{K-1} \eta_{K} \geq 2 \eta_{K}^{2}$.

Below, we use the notation $c_{D}=\sum_{r=D+1}^{\infty} \frac{1}{r^{2}}$ for legibility. The matrices $\Sigma^{Q,(D)}$ and $\Sigma_{\infty}^{Q}$ are symmetric positive definite. By Lemma 1 and the definitions of $\Sigma^{Q,(D)}, \Sigma_{\infty}^{Q}$ in (26) and (23), respectively, we obtain from (34)

$$
\begin{aligned}
& \mathrm{E}\left[\left\|\int_{t}^{t+h} \Psi\left(\int_{t}^{s} \Phi \mathrm{d} W_{r}^{K}\right) \mathrm{d} W_{s}^{K}-\sum_{i, j \in \mathcal{J}_{K}} \hat{I}_{(i, j)}^{Q,(D)}(h) \Psi\left(\Phi \tilde{e}_{i}, \tilde{e}_{j}\right)\right\|_{H}^{2}\right] \\
& \leq \frac{C h^{2} c_{D}}{2 \sqrt{2} \eta_{K} \pi^{2}} \mathrm{E}\left[\left\|\Sigma^{Q,(D)}-\Sigma_{\infty}^{Q}\right\|_{F}^{2}\right] \\
& =\frac{C h^{2} c_{D}}{2 \sqrt{2} \eta_{K} \pi^{2}} \mathrm{E}\left[\| c_{D}^{-1} \sum_{r=D+1}^{\infty} \frac{1}{r^{2}} H_{K} \Sigma^{Q}\left(V_{r}^{Q}\right)_{\mid Z_{r}^{Q}, \Delta w_{h}^{Q}} H_{K}^{T}\right.
\end{aligned}
$$

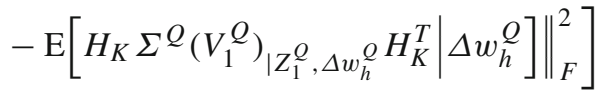

$$
\begin{aligned}
& =\frac{C h^{2} c_{D}}{2 \sqrt{2} \eta_{K} \pi^{2}} \mathrm{E}\left[\| c_{D}^{-1}\left(\sum_{r=D+1}^{\infty} \frac{H_{K} \Sigma^{Q}\left(V_{r}^{Q}\right)_{\mid Z_{r}^{Q}, \Delta w_{h}^{Q}} H_{K}^{T}}{r^{2}}\right.\right. \\
& \left.\left.-\sum_{r=D+1}^{\infty} \frac{\mathrm{E}\left[H_{K} \Sigma^{Q}\left(V_{1}^{Q}\right)_{\mid Z_{1}^{Q}, \Delta w_{h}^{Q}} H_{K}^{T} \mid \Delta w_{h}^{Q}\right]}{r^{2}}\right) \|_{F}^{2}\right] \\
& =\frac{C h^{2} c_{D}^{-1}}{2 \sqrt{2} \eta_{K} \pi^{2}} \sum_{k, l=1}^{L} \mathrm{E}\left[\mathrm { E } \left[\left(\sum_{r=D+1}^{\infty} \frac{{ }_{K} \Sigma^{Q}\left(V_{r}^{Q}\right)_{\mid Z_{r}^{Q}, \Delta w_{h}^{Q} H_{K}^{T}}}{r^{2}}\right.\right.\right.
\end{aligned}
$$

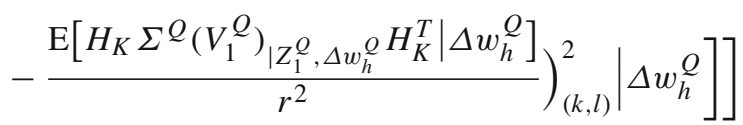

for $h>0, t, t+h \in[0, T], D, K \in \mathbb{N}$. Following ideas from [9], we get

$$
\begin{aligned}
& \mathrm{E}\left[\left\|\int_{t}^{t+h} \Psi\left(\int_{t}^{s} \Phi \mathrm{d} W_{r}^{K}\right) \mathrm{d} W_{s}^{K}-\sum_{i, j \in \mathcal{J}_{K}} \hat{I}_{(i, j)}^{Q,(D)}(h) \Psi\left(\Phi \tilde{e}_{i}, \tilde{e}_{j}\right)\right\|_{H}^{2}\right] \\
& \quad \leq C \frac{h^{2}}{2 \sqrt{2} \eta_{K} \pi^{2}}\left(\sum_{r=D+1}^{\infty} \frac{1}{r^{2}}\right)^{-1}
\end{aligned}
$$




$$
\begin{aligned}
& \times \sum_{k, l=1}^{L} \mathrm{E}\left[\operatorname{Var}\left(\left(\sum_{r=D+1}^{\infty} \frac{1}{r^{2}} H_{K} \Sigma^{Q}\left(V_{r}^{Q}\right)_{\mid Z_{r}^{Q}, \Delta w_{h}^{Q}} H_{K}^{T}\right)_{(k, l)} \mid \Delta w_{h}^{Q}\right)\right] \\
= & C \frac{h^{2}}{2 \sqrt{2} \eta_{K} \pi^{2}}\left(\sum_{r=D+1}^{\infty} \frac{1}{r^{2}}\right)^{-1} \\
& \times \sum_{k, l=1}^{L} \sum_{r=D+1}^{\infty} \frac{1}{r^{4}} \mathrm{E}\left[\operatorname{Var}\left(\left(H_{K} \Sigma^{Q}\left(V_{r}^{Q}\right)_{\mid Z_{r}^{Q}, \Delta w_{h}^{Q}} H_{K}^{T}\right)_{(k, l)} \mid \Delta w_{h}^{Q}\right)\right] .
\end{aligned}
$$

Next, we compute the conditional expectation involved in this estimate. We split the sum into diagonal entries and off-diagonal elements of the matrix, that is,

$$
\begin{aligned}
& \sum_{k, l=1}^{L} \sum_{r=D+1}^{\infty} \frac{1}{r^{4}} \mathrm{E}\left[\operatorname{Var}\left(\left(H_{K} \Sigma^{Q}\left(V_{r}^{Q}\right)_{\mid Z_{r}^{Q}, \Delta w_{h}^{Q}} H_{K}^{T}\right)_{(k, l)} \mid \Delta w_{h}^{Q}\right)\right] \\
& =\sum_{k=1}^{L} \sum_{r=D+1}^{\infty} \frac{1}{r^{4}} \mathrm{E}\left[\mathrm{E}\left[\left(H_{K} \Sigma^{Q}\left(V_{r}^{Q}\right)_{\mid Z_{r}^{Q}, \Delta w_{h}^{Q}} H_{K}^{T}-\Sigma_{\infty}^{Q}\right)_{(k, k)}^{2} \mid \Delta w_{h}^{Q}\right]\right] \\
& \quad+\sum_{\substack{k, l=1 \\
k \neq l}}^{L} \sum_{r=D+1}^{\infty} \frac{1}{r^{4}} \mathrm{E}\left[\mathrm{E}\left[\left(H_{K} \Sigma^{Q}\left(V_{r}^{Q}\right)_{\mid Z_{r}^{Q}, \Delta w_{h}^{Q}} H_{K}^{T}-\Sigma_{\infty}^{Q}\right)_{(k, l)}^{2} \mid \Delta w_{h}^{Q}\right]\right] .
\end{aligned}
$$

Then, we insert the expressions detailed above for $H_{K} \Sigma^{Q}\left(V_{r}^{Q}\right)_{\mid Z_{r}^{Q}, \Delta w_{h}^{Q}} H_{K}^{T}, r \in \mathbb{N}$ and $\Sigma_{\infty}^{Q}$, see (35)-(38). This yields for $h>0, t, t+h \in[0, T], D, L \in \mathbb{N}$

$$
\begin{aligned}
& \sum_{k, l=1}^{L} \sum_{r=D+1}^{\infty} \frac{1}{r^{4}} \mathrm{E}\left[\operatorname{Var}\left(\left(H_{K} \Sigma^{Q}\left(V_{r}^{Q}\right)_{\mid Z_{r}^{Q}, \Delta w_{h}^{Q}} H_{K}^{T}\right)_{(k, l)} \mid \Delta w_{h}^{Q}\right)\right] \\
& =\sum_{\substack{i, j \in \mathcal{J}_{K} \\
i<j}} \sum_{r=D+1}^{\infty} \frac{1}{r^{4}}\left(\mathrm { E } \left[\mathrm { E } \left[\left(\eta_{i}\left(\left(Z_{r j}^{Q}\right)^{2}-2 Z_{r j}^{Q} \sqrt{\frac{2}{h}} \Delta w_{h}^{j}\right)\right.\right.\right.\right. \\
& \left.\left.\left.\left.+\eta_{j}\left(\left(Z_{r i}^{Q}\right)^{2}-2 Z_{r i}^{Q} \sqrt{\frac{2}{h}} \Delta w_{h}^{i}\right)-2 \eta_{i} \eta_{j}\right)^{2} \mid \Delta w_{h}^{Q}\right]\right]\right) \\
& +\sum_{\substack{i, j, m, n \in \mathcal{J}_{K} \\
i<j ; m<n}} \sum_{r=D+1}^{\infty} \frac{1}{r^{4}} \\
& \quad \times\left(\mathrm { E } \left[\mathrm { E } \left[\eta_{i}^{2}\left(Z_{r j}^{Q} Z_{r m}^{Q}-Z_{r j}^{Q} \sqrt{\frac{2}{h}} \Delta w_{h}^{m}-Z_{r m}^{Q} \sqrt{\frac{2}{h}} \Delta w_{h}^{j}\right)^{2} \mathbb{1}_{i=n} \mathbb{1}_{j \neq m}\right.\right.\right. \\
& \quad+\eta_{i}^{2}\left(Z_{r j}^{Q} Z_{r n}^{Q}-Z_{r j}^{Q} \sqrt{\frac{2}{h}} \Delta w_{h}^{n}-Z_{r n}^{Q} \sqrt{\frac{2}{h}} \Delta w_{h}^{j}\right)^{2} \mathbb{1}_{i=m} \mathbb{1}_{j \neq n}
\end{aligned}
$$




$$
\begin{aligned}
& +\eta_{j}^{2}\left(Z_{r i}^{Q} Z_{r m}^{Q}-Z_{r i}^{Q} \sqrt{\frac{2}{h}} \Delta w_{h}^{m}-Z_{r m}^{Q} \sqrt{\frac{2}{h}} \Delta w_{h}^{i}\right)^{2} \mathbb{1}_{j=n} \mathbb{1}_{i \neq m} \\
& \left.\left.\left.+\eta_{j}^{2}\left(Z_{r i}^{Q} Z_{r n}^{Q}-Z_{r i}^{Q} \sqrt{\frac{2}{h}} \Delta w_{h}^{n}-Z_{r n}^{Q} \sqrt{\frac{2}{h}} \Delta w_{h}^{i}\right)^{2} \mathbb{1}_{j=m} \mathbb{1}_{i \neq n} \mid \Delta w_{h}^{Q}\right]\right]\right) .
\end{aligned}
$$

We compute the terms in (39) separately and obtain

$$
\begin{aligned}
\mathrm{E}[\mathrm{E}[ & {\left[\eta_{i}\left(\left(Z_{r j}^{Q}\right)^{2}-2 Z_{r j}^{Q} \sqrt{\frac{2}{h}} \Delta w_{h}^{j}\right)\right.} \\
& \left.\left.\left.+\eta_{j}\left(\left(Z_{r i}^{Q}\right)^{2}-2 Z_{r i}^{Q} \sqrt{\frac{2}{h}} \Delta w_{h}^{i}\right)-2 \eta_{i} \eta_{j}\right)^{2} \mid \Delta w_{h}^{Q}\right]\right] \\
= & \mathrm{E}\left[3 \eta_{i}^{2} \eta_{j}^{2}+2 \eta_{i}^{2} \eta_{j}^{2}-4 \eta_{i}^{2} \eta_{j}^{2}+\frac{8}{h} \eta_{i}^{2} \eta_{j}\left(\Delta w_{h}^{j}\right)^{2}+3 \eta_{i}^{2} \eta_{j}^{2}-4 \eta_{i}^{2} \eta_{j}^{2}\right. \\
& \left.+\frac{8}{h} \eta_{i} \eta_{j}^{2}\left(\Delta w_{h}^{i}\right)^{2}+4 \eta_{i}^{2} \eta_{j}^{2}\right] \\
= & 20 \eta_{i}^{2} \eta_{j}^{2}
\end{aligned}
$$

and

$$
\begin{aligned}
\mathrm{E} & {\left[\mathrm{E}\left[\eta_{i}^{2}\left(Z_{r j}^{Q} Z_{r m}^{Q}-Z_{r j}^{Q} \sqrt{\frac{2}{h}} \Delta w_{h}^{m}-Z_{r m}^{Q} \sqrt{\frac{2}{h}} \Delta w_{h}^{j}\right)^{2} \mathbb{1}_{i=n} \mathbb{1}_{j \neq m} \mid \Delta w_{h}^{Q}\right]\right] } \\
& =\mathrm{E}\left[\eta_{i}^{2}\left(\eta_{j} \eta_{m}+\frac{2}{h} \eta_{j}\left(\Delta w_{h}^{m}\right)^{2}+\frac{2}{h} \eta_{m}\left(\Delta w_{h}^{j}\right)^{2}\right) \mathbb{1}_{i=n} \mathbb{1}_{j \neq m}\right] \\
& =5 \eta_{i}^{2} \eta_{j} \eta_{m} \mathbb{1}_{i=n} \mathbb{1}_{j \neq m}
\end{aligned}
$$

for all $i, j, m, n \in \mathcal{J}_{K}$ with $i<j$ and $m<n$. For the other terms of this type, we get similar results. Moreover, we compute bounds for the following expressions

$$
\sum_{r=D+1}^{\infty} \frac{1}{r^{4}} \leq \int_{D}^{\infty} \frac{1}{s^{4}} \mathrm{~d} s=\frac{1}{3 D^{3}}, \quad \sum_{r=D+1}^{\infty} \frac{1}{r^{2}} \geq \int_{D+1}^{\infty} \frac{1}{s^{2}} \mathrm{~d} s=\frac{1}{D+1}
$$

for all $D \in \mathbb{N}$. A combination of these estimates yields

$$
\left(\sum_{r=D+1}^{\infty} \frac{1}{r^{4}}\right)\left(\sum_{r=D+1}^{\infty} \frac{1}{r^{2}}\right)^{-1} \leq \frac{D+1}{3 D^{3}} \leq \frac{2}{3 D^{2}}
$$

for all $D \in \mathbb{N}$. At this point, the main difference to Algorithm 1 arises-we obtain a higher order of convergence in $D$. In total, we get 


$$
\begin{aligned}
\mathrm{E}\left[\left\|\int_{t}^{t+h} \Psi\left(\Phi \int_{t}^{s} \mathrm{~d} W_{r}^{K}\right) \mathrm{d} W_{s}^{K}-\sum_{i, j \in \mathcal{J}_{K}} \hat{I}_{(i, j)}^{Q,(D)}(h) \Psi\left(\Phi \tilde{e}_{i}, \tilde{e}_{j}\right)\right\|_{H}^{2}\right] \\
\leq C \frac{h^{2}}{2 \sqrt{2} \eta_{K} \pi^{2}}\left(\sum_{r=D+1}^{\infty} \frac{1}{r^{2}}\right)^{-1} \sum_{\substack{r=D+1 \\
r^{4}}}^{\infty} \frac{1}{\substack{i, j \in \mathcal{J}_{K} \\
i<j}} 20 \eta_{i}^{2} \eta_{j}^{2} \\
\quad+\sum_{\substack{i, j, m, n \in \mathcal{J}_{K} \\
i<j ; m<n}} 5\left(\eta_{i}^{2} \eta_{j} \eta_{m} \mathbb{1}_{i=n} \mathbb{1}_{j \neq m}+\eta_{i}^{2} \eta_{j} \eta_{n} \mathbb{1}_{i=m} \mathbb{1}_{j \neq n}\right. \\
\left.\left.\quad+\eta_{j}^{2} \eta_{i} \eta_{m} \mathbb{1}_{j=n} \mathbb{1}_{i \neq m}+\eta_{j}^{2} \eta_{i} \eta_{n} \mathbb{1}_{j=m} \mathbb{1}_{i \neq n}\right)\right) \\
\leq C \frac{h^{2}}{2 \sqrt{2} \eta_{K} \pi^{2}} \frac{2}{3 D^{2}} \sum_{\substack{i, j \in \mathcal{J}_{K} \\
i<j}}\left(20 \eta_{i}^{2} \eta_{j}^{2}+10 \eta_{i}^{2} \eta_{j} \sum_{\substack{m \in \mathcal{J}_{K} \\
m \neq j}} \eta_{m}+10 \eta_{j}^{2} \eta_{i} \sum_{\substack{m \in \mathcal{J}_{K} \\
m \neq i}} \eta_{m}\right) .
\end{aligned}
$$

Finally, this implies for all $h>0, t, t+h \in[0, T], D, K \in \mathbb{N}$

$$
\begin{aligned}
& \mathrm{E}\left[\left\|\int_{t}^{t+h} \Psi\left(\Phi \int_{t}^{s} \mathrm{~d} W_{r}^{K}\right) \mathrm{d} W_{s}^{K}-\sum_{i, j \in \mathcal{J}_{K}} \hat{I}_{(i, j)}^{Q,(D)}(h) \Psi\left(\Phi \tilde{e}_{i}, \tilde{e}_{j}\right)\right\|_{H}^{2}\right] \\
& \leq C \frac{h^{2}}{2 \sqrt{2} \eta_{K} \pi^{2}} \frac{2}{3 D^{2}}\left(20\left(\max _{j \in \mathcal{J}_{K}} \eta_{j}\right)^{2}(\operatorname{tr} Q)^{2}+20\left(\max _{j \in \mathcal{J}_{K}} \eta_{j}\right)(\operatorname{tr} Q)^{3}\right) \\
& \leq C \max _{j \in \mathcal{J}_{K}} \eta_{j}(\operatorname{tr} Q)^{3} \frac{h^{2}}{\eta_{K} D^{2}},
\end{aligned}
$$

that is, more generally,

$$
\begin{aligned}
& \mathrm{E}\left[\left\|\int_{t}^{t+h} \Psi\left(\Phi \int_{t}^{s} \mathrm{~d} W_{r}^{K}\right) \mathrm{d} W_{s}^{K}-\sum_{i, j \in \mathcal{J}_{K}} \hat{I}_{(i, j)}^{Q,(D)}(h) \Psi\left(\Phi \tilde{e}_{i}, \tilde{e}_{j}\right)\right\|_{H}^{2}\right] \\
& \leq C \max _{j \in \mathcal{J}_{K}} \eta_{j}(\operatorname{tr} Q)^{3} \frac{h^{2}}{\left(\min _{j \in \mathcal{J}_{K}} \eta_{j}\right) D^{2}} .
\end{aligned}
$$

The statement of the theorem follows by combing this estimate with (30).

Acknowledgements Funding and support by the Graduate School for Computing in Medicine and Life Sciences funded by Germany's Excellence Initiative [DFG GSC 235/2] and in addition by the Cluster of Excellence "The Future Ocean" is gratefully acknowledged. "The Future Ocean" is funded within the framework of the Excellence Initiative by the Deutsche Forschungsgemeinschaft (DFG) on behalf of the German federal and state governments.

Open Access This article is distributed under the terms of the Creative Commons Attribution 4.0 International License (http://creativecommons.org/licenses/by/4.0/), which permits unrestricted use, distribution, and reproduction in any medium, provided you give appropriate credit to the original author(s) and the source, provide a link to the Creative Commons license, and indicate if changes were made. 


\section{References}

1. Da Prato, G., Zabczyk, J.: Stochastic equations in infinite dimensions, 2nd edn. Cambridge University Press, Cambridge (2014).https://doi.org/10.1017/CBO9781107295513

2. Jentzen, A., Röckner, M.: A Milstein scheme for SPDEs. Found. Comput. Math. 15(2), 313-362 (2015). https://doi.org/10.1007/s10208-015-9247-y

3. Kloeden, P.E., Platen, E.: Numerical solution of stochastic differential equations, Second corrected printing edn. Springer, Berlin (1995). http://dx.doi.org/10.1007/978-3-662-12616-5

4. Kloeden, P.E., Platen, E., Wright, I.W.: The approximation of multiple stochastic integrals. Stoch. Anal. Appl. 10(4), 431-441 (1992). https://doi.org/10.1080/07362999208809281

5. Leonhard, C.: Derivative-free numerical schemes for stochastic partial differential equations. Ph.D. thesis, Institute of Mathematics, Universität zu Lübeck (2016)

6. Leonhard, C., Rößler, A.: Enhancing the order of the Milstein scheme for stochastic partial differential equations with commutative noise. SIAM J. Numer. Anal. 56(4), 2585-2622 (2018). https://doi.org/ $10.1137 / 16 \mathrm{M} 1094087$

7. Milstein, G.N.: Numerical integration of stochastic differential equations. Kluwer Academic Publishers, Dordrecht (1995). https://doi.org/10.1007/978-94-015-8455-5. [Translated and revised from the 1988 Russian original]

8. Prévôt, C., Röckner, M.: A Concise Course on Stochastic Partial Differential Equations. Lecture Notes in Mathematics, vol. 1905. Springer, Berlin (2007)

9. Wiktorsson, M.: Joint characteristic function and simultaneous simulation of iterated Itô integrals for multiple independent Brownian motions. Ann. Appl. Probab. 11(2), 470-487 (2001). https://doi.org/ 10.1214/aoap/1015345301

10. Wong, E., Zakai, M.: On the convergence of ordinary integrals to stochastic integrals. Ann. Math. Stat. 36(5), 1560-1564 (1965)

11. Wong, E., Zakai, M.: On the relation between ordinary and stochastic differential equations. Int. J. Eng. Sci. 3, 213-229 (1965) 